\title{
Istituto
}

Nazionale

Sezione di ROMA

Piazzale Aldo Moro, 2

I-00185 Roma, Italy

Fisica

INFN-1296/00

Nucleare

June 2000

\section{Poincaré Covariant Current Operator and Elastic Electron-Deuteron Scattering in the Front-form Hamiltonian Dynamics}

\author{
F.M. Lev ${ }^{a}$, E. Pace ${ }^{b}$ and G. Salmè ${ }^{c}$ \\ ${ }^{a}$ Laboratory of Nuclear Problems, Joint Institute for Nuclear Research, Dubna, Moscow re- \\ gion 141980, Russia \\ ${ }^{b}$ Dipartimento di Fisica, Università di Roma "Tor Vergata", and Istituto Nazionale di Fisica \\ Nucleare, Sezione Tor Vergata, Via della Ricerca Scientifica 1, I-00133, Rome, Italy \\ ${ }^{c}$ Istituto Nazionale di Fisica Nucleare, Sezione di Roma, P.le A. Moro 2, I-00185 Rome, \\ Italy
}

\begin{abstract}
The deuteron electromagnetic form factors, $A\left(Q^{2}\right)$ and $B\left(Q^{2}\right)$, and the tensor polarization $T_{20}\left(Q^{2}\right)$, are unambiguously calculated within the front-form relativistic Hamiltonian dynamics, by using a novel current, built up from one-body terms, which fulfills Poincaré, parity and time reversal covariance, together with Hermiticity and the continuity equation. A simultaneous description of the experimental data for the three deuteron form factors is achieved up to $Q^{2}<0.4(\mathrm{GeV} / \mathrm{c})^{2}$. At higher momentum transfer, different nucleon-nucleon interactions strongly affect $A\left(Q^{2}\right), B\left(Q^{2}\right)$, and $T_{20}\left(Q^{2}\right)$ and the effects of the interactions can be related to $S$-state kinetic energy in the deuteron. Different nucleon form factor models have huge effects on $A\left(Q^{2}\right)$, smaller effects on $B\left(Q^{2}\right)$ and essentially none on $T_{20}\left(Q^{2}\right)$.
\end{abstract}

Submitted to Phys. Rev. C 


\section{Introduction}

The deuteron is a fundamental system for our understanding of nuclear physics and a challenge to our ability to describe nuclei as systems of interacting nucleons with a well defined internal structure, without an explicit use of their quark substructure. In particular elastic electron-deuteron scattering is a crucial test for deuteron models.

There exists a wide literature (see, e.g., [1, 2, 3, 6, 6, 6, 7] and Refs. quoted therein) devoted to the investigation of deuteron electromagnetic (em) properties and in particular to the accuracy of the one-body impulse approximation (IA) for the current operator. It is usually believed that effects beyond IA, as meson-exchange currents, $N \bar{N}$-pair creation terms ( $Z$ graphs), isobar configurations in the deuteron wavefunction, etc. are important for the explanation of existing data. However, the contributions of these effects are essentially model dependent [8] and, furthermore, obviously depend on the reference frame (see, e.g., Refs. [9, 10]).

Since precise measurements of the deuteron elastic form factors have been recently performed in a wide range of momentum transfer, up to $Q^{2}=-q_{\mu}^{2}=6(\mathrm{GeV} / \mathrm{c})^{2}$ for $A\left(Q^{2}\right)$ [11, [1], theoretical models require a relativistic framework for a reliable description of the available data. Furthermore, it has been recently shown [13] that relativistic effects are relevant even for static deuteron properties, as the magnetic and quadrupole moments.

An essential requirement for relativistic approaches is the covariance of the current operator with respect to Poincaré group transformations. This requirement is non-trivial for systems of interacting particles, since some of the generators are interaction dependent.

A widely adopted relativistic framework for the study of deuteron em properties is the front-form Hamiltonian dynamics (FFHD) with a finite number of particles (see Refs. [14, 15] and Refs. [16, 17] for extensive reviews), which gives the possibility to retain the large amount of successfull phenomenology developed within the nonrelativistic approaches. Indeed, in the FFHD seven, out of ten, Poincaré generators are interaction free, in particular the boost generators, while $P^{-}=\left(P_{0}-P_{z}\right) / \sqrt{2}(P$ is the total momentum of the system) and the rotations around the $x$ and $y$ axes contain the dynamics. Only the two-nucleon state is usually considered and the wavefunction of the system factorizes for any front-form boost in an eigenfunction of the total momentum times an intrinsic wavefunction, depending only on internal variables. Therefore, in the case of elastic $e-d$ scattering, one can express the three deuteron em form factors, determined by three independent matrix elements of the current, in terms of the deuteron internal wave function and the elastic em nucleon form factors (f.f.).

In the FFHD the em properties of the deuteron were usually studied in the reference frame where $q^{+}=\left(q_{0}+q_{z}\right) / \sqrt{2}=0$ ( $q$ is the momentum transfer) [1, 9, 18, 19, 20, 21, 22]. The one-body approximation was used to define three matrix elements of the plus component of the current, while the other matrix elements of the plus component and the other components of the current were properly defined in order to fulfill Poincaré covariance, Hermiticity and current conservation. However, for spin-one systems, as the deuteron, this procedure is not unique and gives rise to ambiguities in the calculation of the form factors [18, 23]. 
In Ref. [10], using a representation of the Poincaré group within FFHD, we have shown that extended Poincaré covariance (i.e., Poincaré plus parity, $\mathcal{P}$, and time reversal, $\mathcal{T}$, covariance) is fulfilled by the current which has a one-body form in the Breit reference frame where the initial and final momenta of the system are directed along the spin quantization axis $\left(\vec{q}_{\perp}=\vec{q}-q_{z} \boldsymbol{e}_{z}=0\right)$. Furthermore, we have shown that Hermiticity and current conservation can be easily implemented. An important feature of our approach is that it allows one to use the same definition for all the matrix elements of the current, without ambiguities.

In a previous paper [13], as a test of our current, we evaluated the deuteron form factors at $Q^{2}=0$, namely the magnetic moment, $\mu_{d}$, and the quadrupole moment, $Q_{d}$, of the deuteron, which are not affected by the uncertainties in the knowledge of the neutron em form factors at finite momentum transfers. The deuteron magnetic and quadrupole moments represented a longstanding problem in nuclear physics. Indeed, theoretical calculations were not able to accurately reproduce in a coherent approach the experimental values for both quantities at the same time, although a variety of approaches have been attempted, by changing the tensor content of the nucleon-nucleon $(N-N)$ interaction, or considering twobody current contributions, both in non-relativistic and in relativistic frameworks [24, 25, 26, 51. On the contrary, using our Poincaré covariant current operator, this usual disagreement between theoretical and experimental results was reduced to $0.5 \%$ for $\mu_{d}$ and to $2 \%$ for $Q_{d}$ by using interactions able to reproduce the experimental value of the deuteron asymptotic normalization ratio $\eta=A_{D} / A_{S}$. Therefore the contributions from explicit two-body currents or from isobar configurations in the deuteron wave function should be relatively small at $Q^{2}=0$.

Encouraged by this result, in the present paper we study, within the framework of FFHD and using our Poincaré covariant current operator, the deuteron form factors at $Q^{2} \neq 0$ and in particular the effects produced by : i) different $N-N$ interactions, and ii) different nucleon form factors models. We will also investigate the possibility to gain information from elastic $e-d$ scattering on the neutron em structure, and in particular on the neutron charge form factor. Our preliminary results were already published in Ref. [27].

The plan of the paper is the following: in Sect. 2 the definition of our covariant current operator is recalled; in Sect. 3 the elastic deuteron form factors are expressed in terms of the matrix elements of the free current in the Breit frame; in Sect. 4 the front-form deuteron wave function and the explicit expressions of the current matrix elements in terms of the deuteron wave function are presented; in Sect. 5 our results on the dependence of deuteron form factors upon $N-N$ interactions and nucleon em form factors are discussed and, eventually, in Sect. 6 our conclusions are drawn. 


\section{A covariant current operator within the front-form dynamics}

In this section we give the essential lines for the definition of a current which satisfies extended Poincaré covariance, Hermiticity, current conservation, and charge normalization, to be applied to the calculation of elastic em form factors.

Let us first consider the extended Poincaré covariance. If the current operator $J^{\mu}(x)$ is defined in terms of $J^{\mu}(0)$

$$
J^{\mu}(x)=\exp (\imath P x) J^{\mu}(0) \exp (-\imath P x),
$$

then the Poincaré covariance of $J^{\mu}(x)$ takes place if

$$
U(l)^{-1} J^{\mu}(0) U(l)=L(l)_{\nu}^{\mu} J^{\nu}(0),
$$

where $L(l)$ is the element of the Lorentz group corresponding to $l \in S L(2, C)$ and $U(l)$ is the unitary representation operator corresponding to $l$ (see, e.g., [28]).

For systems of interacting particles the operator $U(l)$ in general does depend on the interaction, and it is not trivial to build up a current which satisfies Eq. (2). Indeed, in order to fulfill this requirement the current operator has to be interaction dependent. The key property of our procedure [10] for the definition of a Poincaré covariant current operator is the following spectral decomposition of the current:

$$
J^{\mu}(0)=\sum_{i j} \Pi_{i} J^{\mu}(0) \Pi_{j}
$$

In Eq. (3) $\Pi_{i}$ is the orthogonal projector onto the subspace $\mathcal{H}_{i} \equiv \Pi_{i} \mathcal{H}$ corresponding to the mass $M_{i}$, the spin $S_{i}$, and a definite parity, with $\mathcal{H}$ being the space of states describing the interacting particle system. This decomposition allows one to express the possible current operator dependence on the interaction as a dependence on mass and spin of the interacting particle system.

In the FFHD, the seven Poincaré generators belonging to the subgroup which leaves invariant the hyperplane $x^{+}=0$ are kinematical. Then, as already mentioned in the introduction, the state of a system, $|P, \chi\rangle$, factorizes in a total momentum eigenstate, $\left|\vec{P}_{\perp}, P^{+}\right\rangle$, times an intrinsic eigenstate, $|\chi\rangle$ :

$$
|P, \chi\rangle=\left|\vec{P}_{\perp}, P^{+}\right\rangle|\chi\rangle
$$

In Eq. (14) $P^{+}=\left(P_{0}+P_{z}\right) / \sqrt{2}=p_{1}^{+}+\ldots+p_{N}^{+}$and $\vec{P}_{\perp}=\left(P_{x}, P_{y}\right)=\vec{p}_{1 \perp}+\ldots+\vec{p}_{N \perp}$ are the plus and $\perp$ components of the total momentum, with $p_{1}, \ldots, p_{N}$ the individual momenta of the particles in the system. Because of the decomposition of Eq. (3), the operator $J^{\mu}(0)$ is fully defined by the set of matrix elements between initial, $\left|\boldsymbol{P}_{\perp}, P_{j}^{+}\right\rangle$, and final, $\left|\boldsymbol{P}_{\perp}^{\prime}, P_{i}^{\prime+}\right\rangle$, total momentum eigenstates

$$
J^{\mu}\left(P_{i}^{\prime} ; P_{j}\right) \equiv\left\langle\boldsymbol{P}_{\perp}^{\prime}, P_{i}^{\prime}{ }^{\prime}\left|\Pi_{i} J^{\mu}(0) \Pi_{j}\right| \boldsymbol{P}_{\perp}, P_{j}^{+}\right\rangle
$$


The matrix elements between total momentum eigenstates, $J^{\mu}\left(P_{i}^{\prime} ; P_{j}\right)$, correspond to definite values of masses, spins and parity, and are operators in the space $\mathcal{H}_{\text {int }}$ of intrinsic states. Through proper unitary transformations, the current operator $J^{\mu}\left(P_{i}^{\prime}, P_{j}\right)$ in any reference frame can be defined in terms of the auxiliary current operators

$$
j^{\nu}\left(K \boldsymbol{e}_{z} ; M_{i}, M_{j}\right) \equiv\left\langle\boldsymbol{K}_{i \perp}^{\prime}=0, K_{i}^{\prime}\left|\Pi_{i} J^{\mu}(0) \Pi_{j}\right| \boldsymbol{K}_{j \perp}=0, K_{j}^{+}\right\rangle
$$

in the special Breit frame where the total three-momenta of the system in the initial state, $\boldsymbol{K}_{j}=-K \boldsymbol{e}_{z}$, and in the final state, $\boldsymbol{K}_{i}^{\prime}=K \boldsymbol{e}_{z}$, are directed along the spin quantization axis, z. In Eq. (6) the initial and final plus components of the total momentum are

$$
K_{j}^{+}=\frac{1}{\sqrt{2}}\left[\left(M_{j}+K^{2}\right)^{1 / 2}-K\right], \quad K_{i}^{\prime+}=\frac{1}{\sqrt{2}}\left[\left(M_{i}^{2}+K^{2}\right)^{1 / 2}+K\right],
$$

while $K=Q / 2$, and $q=K_{i}^{\prime}-K_{j}$. It has been shown [10 that the operator $J^{\mu}(0)$ fulfills Eq. (2), i.e. is Lorentz covariant, if the current operators $j^{\nu}\left(K \boldsymbol{e}_{z} ; M_{i}, M_{j}\right)$ in the above special Breit frame are covariant with respect to rotations around the $z$ axis.

Since in the front form the rotations around the $z$ axis are interaction free, the continuous Lorentz transformations constrain the current $j^{\mu}\left(K \boldsymbol{e}_{z} ; M_{i}, M_{j}\right)$ for an interacting system in the same way as in the non-interacting case. The same property holds for the covariance with respect to a reflection of the $y$ axis, $\mathcal{P}_{y}$, and with respect to the product of parity and time reversal, $\theta$, which leave the light cone $x^{+}=0$ invariant, and therefore are kinematical. The full space reflection is the product of $\mathcal{P}_{y}$ and a dynamical rotation around the $y$ axis by $\pi$, while $\mathcal{T}=\theta \mathcal{P}$, and therefore parity and time reversal do not contain an interaction dependence different from the one implied by rotations around $y$ axis. As a consequence, the current operator satisfies $\mathcal{P}$ and $\mathcal{T}$ covariance, if it satisfies Poincaré covariance and covariance with respect to $P_{y}$ and $\theta[10$.

In conclusion, since in our Breit frame the extended Poincaré covariance constraints for the auxiliary operators are the same for a non-interacting and an interacting system, the extended Poincaré covariance is satisfied for an interacting system by a current composed by the sum of free, one-body currents, viz.

$$
j_{\text {free }}^{\mu}\left(K \boldsymbol{e}_{z} ; M_{i}, M_{j}\right) \equiv\left\langle 0, K_{i}^{+}\left|\Pi_{i} J_{\text {free }}^{\mu}(0) \Pi_{j}\right| 0, K_{j}^{+}\right\rangle
$$

where $J_{\text {free }}^{\mu}(0)=\sum_{i=1}^{N} j_{\text {free }, i}^{\mu}$, with $\mathrm{N}$ the number of constituents in the system.

In the elastic case considered in this paper $\left(M_{i}=M_{j}=M ; S_{i}=S_{j}=S\right)$, the property of Hermiticity for the auxiliary operators reads as follows

$$
j^{\mu}(-\boldsymbol{K} ; M, M)=j^{\mu}(\boldsymbol{K} ; M, M)^{*},
$$

where ${ }^{*}$ means the Hermitian conjugation in the internal space $\mathcal{H}_{\text {int }}$. For $|\boldsymbol{K}|=0$ the property of Hermiticity reads $j^{\mu}(0 ; M, M)=j^{\mu}(0 ; M, M)^{*}$, while for $|\boldsymbol{K}| \neq 0$ it becomes a non-trivial constraint and is satisfied if

$$
\begin{aligned}
& j^{\mu}\left(K \boldsymbol{e}_{z} ; M, M\right)^{*}= \\
& \left.L\left[r_{x}(-\pi)\right]_{\nu}^{\mu} D^{S}\left[r_{x}(-\pi)\right] j^{\nu}\left(K \boldsymbol{e}_{z} ; M, M\right) D^{S}\left[r_{x}(-\pi)\right]\right)^{-1},
\end{aligned}
$$


where $D^{s}(u)$ is the matrix of the unitary irreducible representation of the group $S U(2)$ with spin $s$, corresponding to $u \in S U(2)$, and $r_{x}(-\pi)$ represents a rotation by $-\pi$ around the $x$ axis, i.e. $D^{S}\left[r_{x}(-\pi)\right]=\exp \left(\imath \pi S_{x}\right)[10$.

Let $\Pi$ be the projector onto the subspace of bound states $|\chi\rangle$ of mass $M$ and spin $S$, and let $\mathcal{J}^{\mu}\left(K \boldsymbol{e}_{z} ; M, M\right)$ be a current which fulfills extended Poincaré covariance. Then a choice for the current compatible with the Hermiticity condition, Eq. (10), and with the extended Poincaré covariance is [10]

$$
\begin{array}{r}
j^{\mu}\left(K \boldsymbol{e}_{z} ; M, M\right)=\frac{1}{2}\left\{\mathcal{J}^{\mu}\left(K \boldsymbol{e}_{z} ; M, M\right)+\right. \\
\left.L_{\nu}^{\mu}\left[r_{x}(-\pi)\right] \exp \left(\imath \pi S_{x}\right)\left[\mathcal{J}^{\nu}\left(K \boldsymbol{e}_{z} ; M, M\right)\right]^{*} \exp \left(-\imath \pi S_{x}\right)\right\} .
\end{array}
$$

The second term in Eq. (11), which ensures Hermiticity, introduces implicitely two-body terms in the current, because of the presence of the $x$ component of the front-form spin operator, $S_{x}$.

This current fulfills also the current conservation, which in the elastic case reads

$$
j^{-}\left(K \boldsymbol{e}_{z} ; M, M\right)=j^{+}\left(K \boldsymbol{e}_{z} ; M, M\right)
$$

Indeed, as shown in ref. [10], in the elastic case the extended Poincaré covariance and Hermiticity imply Eq. (12), i.e., impose current conservation.

In Eq. (11) one has to choose a specific definition for the operator $\mathcal{J}^{\mu}\left(K \boldsymbol{e}_{z} ; M, M\right)$. Unfortunately, one cannot simply adopt Eq. (8), because of the charge normalization condition, which implies

$$
j^{+}(0 ; M, M)=\frac{1}{2}\left\{\mathcal{J}^{+}(0 ; M, M)+\mathcal{J}^{-}(0 ; M, M)\right\}=\sqrt{2} e M \Pi .
$$

where $e$ is the total electric charge of the system. Indeed, while the charge normalization condition is fulfilled by $j_{\text {free }}^{+}(0 ; M, M)$, Eq. (13) is not satisfied by $\frac{1}{2}\left(j_{\text {free }}^{+}(0 ; M, M)+\right.$ $\left.j_{\text {free }}^{-}(0 ; M, M)\right)$. However, a possible choice is the following one:

$$
\begin{array}{r}
\mathcal{J}^{+}\left(K \boldsymbol{e}_{z} ; M, M\right)=\left\langle 0, K^{\prime}\left|\Pi J_{\text {free }}^{+}(0) \Pi\right| 0, K^{+}\right\rangle \\
\mathcal{J}_{\perp}\left(K \boldsymbol{e}_{z} ; M, M\right)=\left\langle 0, K^{\prime+}\left|\Pi \boldsymbol{J}_{\perp \text { free }}(0) \Pi\right| 0, K^{+}\right\rangle \\
\mathcal{J}^{-}\left(K \boldsymbol{e}_{z} ; M, M\right)=\mathcal{J}^{+}\left(K \boldsymbol{e}_{z} ; M, M\right)
\end{array}
$$

The previous definition of the "-" component of $\mathcal{J}^{\mu}$ is essential for the proper charge normalization of $j^{\mu}\left(K \boldsymbol{e}_{z} ; M, M\right)$, because of the second term in Eqs. (11,13).

In the elastic case, only $2 S+1$ non-zero matrix elements of the em current defined by Eqs. (11,14) are independent, corresponding to the $2 S+1$ elastic form factors. The independent matrix elements can be chosen as the diagonal matrix elements of $j^{+}$with $S_{z} \geq 0$ and the matrix elements $\left\langle M S S_{z}\left|j_{x}\left(K \boldsymbol{e}_{z} ; M, M\right)\right| M S S_{z}-1\right\rangle$ of $j_{x}$ with $S_{z} \geq+1 / 2$ [10]. Obviously, any other choice of the independent matrix elements to be used in the 
calculation of the elastic form factors is completely equivalent, i.e. it will yield exactly the same results. One can immediately obtain that

$$
\begin{aligned}
& \left\langle M S S_{z}\left|j^{+}\left(K \boldsymbol{e}_{z} ; M, M\right)\right| M S S_{z}\right\rangle=\left\langle M S S_{z}\left|\mathcal{J}^{+}\left(K \boldsymbol{e}_{z} ; M, M\right)\right| M S S_{z}\right\rangle, \\
& \left\langle M S S_{z}\left|j_{x}\left(K \boldsymbol{e}_{z} ; M, M\right)\right| M S S_{z}^{\prime}\right\rangle=\frac{1}{2}\left[\left\langle M S S_{z}\left|\mathcal{J}_{x}\left(K \boldsymbol{e}_{z} ; M, M\right)\right| M S S_{z}^{\prime}\right\rangle-\right. \\
& \left.\left\langle M S S_{z}^{\prime}\left|\mathcal{J}_{x}\left(K \boldsymbol{e}_{z} ; M, M\right)\right| M S S_{z}\right\rangle\right]
\end{aligned}
$$

and therefore the elastic form factors can be evaluated in terms of the matrix elements of the free current only. It has to be noted that the matrix elements of both $j^{+}$and $j_{x}$ have been shown to be real 10 .

In the deuteron case, since $S=1$, three matrix elements of the current are needed.

\section{Deuteron electromagnetic form factors}

The form factors $A\left(Q^{2}\right)$ and $B\left(Q^{2}\right)$, which appear in the unpolarized cross section, and the tensor polarization, $T_{20}\left(Q^{2}\right)$, can be expressed in terms of the charge, $G_{C}\left(Q^{2}\right)$, quadrupole, $G_{Q}\left(Q^{2}\right)$, and magnetic, $G_{M}\left(Q^{2}\right)$, elastic form factors :

$$
\begin{aligned}
& A\left(Q^{2}\right)=G_{C}^{2}+\frac{8}{9} \tau^{2} G_{Q}^{2}+\frac{2}{3} \tau G_{M}^{2} \\
& B\left(Q^{2}\right)=\frac{4}{3} \tau(1+\tau) G_{M}^{2} \\
& T_{20}\left(Q^{2}\right)=-\tau \frac{\sqrt{2}}{3} \frac{\left[\frac{4}{3} \tau G_{Q}^{2}+4 G_{Q} G_{C}+f G_{M}^{2}\right]}{A+B \tan ^{2}(\theta / 2)}
\end{aligned}
$$

where $\tau=Q^{2} /\left(4 m_{d}^{2}\right), Q^{2}=-q_{\mu}^{2}, m_{d}$ is the deuteron mass and $f=1 / 2+(1+\tau) \tan ^{2}(\theta / 2)$, with the following normalization for the form factors: $G_{C}(0)=1, G_{Q}(0)=m_{d}^{2} Q_{d}$, and $G_{M}(0)=\mu_{d} m_{d} / m_{p}\left(m_{p}\right.$ is the proton mass).

For the deuteron, the matrix elements of the current are related to the form factors $G_{C}\left(Q^{2}\right), G_{M}\left(Q^{2}\right), G_{Q}\left(Q^{2}\right)$ by the following general expression of the macroscopic current for spin 1 systems (as the deuteron) 29

$$
\begin{aligned}
& j_{S_{z}^{\prime}, S_{z}}^{\mu}=\left\langle m_{d} 1 S_{z}^{\prime}\left|j^{\mu}\left(K \vec{e}_{z}, m_{d}, m_{d}\right)\right| m_{d} 1 S_{z}\right\rangle=e e_{S_{z}^{\prime}}^{* \alpha} e_{S_{z}}^{\beta}\left\{( P + P ^ { \prime } ) ^ { \mu } \left[-\left(G_{C}-\frac{2}{3} \tau G_{Q}\right) g_{\alpha \beta}\right.\right. \\
& \left.\left.-\zeta^{2}\left[G_{C}-\left(1+\frac{2}{3} \tau\right) G_{Q}-G_{M}\right] q_{\alpha} q_{\beta}\right]+G_{M}\left(g_{\alpha}^{\mu} q_{\beta}-g_{\beta}^{\mu} q_{\alpha}\right)\right\}
\end{aligned}
$$

where $\left|m_{d} 1 S_{z}\right\rangle$ is the deuteron intrinsic eigenstate, $g_{\alpha \beta}$ the metric tensor, $e_{S_{z}}$ and $e_{S_{z}^{\prime}}^{\prime}$ are the initial and final deuteron polarization vectors, respectively, (see Appendix A) and $\zeta^{-1}=$ $\sqrt{2} m_{d} \sqrt{1+\tau}$. 
In FFHD, hadron form factors are often calculated in the reference frame where $q^{+}=$ 0 . If $\lambda$ and $\lambda^{\prime}$ are the helicities in the initial and final states, respectively, and $I_{\lambda^{\prime} \lambda}=$ $\left\langle\lambda^{\prime}\left|J^{+}(0)\right| \lambda\right\rangle$, then, because of Hermiticity, $\mathcal{P}$ and $\mathcal{T}$ covariance, and covariance for rotations about the $z$ axis, all the matrix elements $I_{\lambda^{\prime} \lambda}$ for the deuteron can be expressed in terms of $I_{11}, I_{00}, I_{10}$ and $I_{1,-1}$. As shown, e.g., in Refs. [18, 9], the following constraint, usually called "angular condition", must be fulfilled in the $q^{+}=0$ frame, viz.

$$
(1+2 \tau) I_{11}-I_{00}-(8 \tau)^{1 / 2} I_{10}+I_{1,-1}=0 .
$$

However, this constraint, which is related to the rotational covariance of the current, is not satisfied if the matrix elements $I_{\lambda^{\prime} \lambda}$ are calculated with the free operator, $J_{\text {free }}^{+}(0)$ in the $q^{+}=0$ frame. Then, three out of the four matrix elements are usually defined through the free operator, while the fourth one is defined by Eq. (19). However, different choices of the three matrix elements to be calculated by the free operator are possible and therefore different prescriptions can be used to calculate the three physical form factors. As a consequence, within this approach there is a large ambiguity in the theoretical results (see, e.g., [9, 18, 19, 20, 21, 23), and, furthermore, different definitions are used for different matrix elements of the current.

A relevant result of our approach is that, using in the left hand side of Eq. (18) the microscopic current defined by Eqs. (11, 14), the extraction of elastic em form factors is no more plagued by the ambiguities, which are present when the free current is used in the reference frame where $q^{+}=0$. Indeed, using our current operator, it turns out that only three matrix elements $j_{S_{z}^{\prime}, S_{z}}^{\mu}$ are independent, corresponding to the three elastic em form factors. For instance, one can consider the matrix elements $j_{0,0}^{+}, j_{1,1}^{+}, j_{1,0}^{x}$, which have been shown to be real [10]. On the contrary, using the one-body current in the $q^{+}=0$ frame, one has four independent matrix elements [18].

The form factors $G_{C}, G_{M}$, and $G_{Q}$ can be easily obtained from the matrix elements of the current in our Breit frame, since from Eq. (18) one has

$$
\begin{aligned}
\left\langle m_{d} 11\left|j^{+}\left(K \boldsymbol{e}_{z} ; m_{d}, m_{d}\right)\right| m_{d} 11\right\rangle & =\zeta^{-1}\left[G_{C}-\frac{2}{3} \tau G_{Q}\right] \\
\left\langle m_{d} 10\left|j^{+}\left(K \boldsymbol{e}_{z} ; m_{d}, m_{d}\right)\right| m_{d} 10\right\rangle & =\zeta^{-1}\left[G_{C}+\frac{4}{3} \tau G_{Q}\right] \\
\left\langle m_{d} 11\left|j_{x}\left(K \boldsymbol{e}_{z} ; m_{d}, m_{d}\right)\right| m_{d} 10\right\rangle & =\zeta^{-1} \tau^{\frac{1}{2}} G_{M}
\end{aligned}
$$

By means of Eq. (20) and using the properties (15), (16) of the matrix elements $j_{S_{z}^{\prime}, S_{z}}^{\mu}$, the form factors $G_{C}, G_{M}$, and $G_{Q}$ can be expressed in terms of the matrix elements $\mathcal{J}_{S_{z}, S_{z}}^{+}=\left\langle m_{d} 1 S_{z}\left|\mathcal{J}^{+}\left(K \vec{e}_{z}, m_{d}, m_{d}\right)\right| m_{d} 1 S_{z}\right\rangle$ and $\mathcal{J}_{S_{z}^{\prime}, S_{z}}^{x}=\left\langle m_{d} 1 S_{z}^{\prime}\left|\mathcal{J}_{x}\left(K \vec{e}_{z}, m_{d}, m_{d}\right)\right| m_{d} 1 S_{z}\right\rangle$, i.e. in terms of the matrix elements of the free current, calculated in the Breit frame where the momentum transfer is along the spin quantization axis, $z$ [27]. One obtains

$$
G_{C}=\left(2 \mathcal{J}_{1,1}^{+}+\mathcal{J}_{0,0}^{+}\right) \zeta / 3, \quad G_{M}=\left(\mathcal{J}_{1,0}^{x}-\mathcal{J}_{0,1}^{x}\right) \zeta /(2 \sqrt{\tau}), \quad G_{Q}=\left(\mathcal{J}_{0,0}^{+}-\mathcal{J}_{1,1}^{+}\right) \zeta /(2 \tau) .
$$


Then, the deuteron magnetic moment, in nuclear magnetons, is given by

$$
\mu_{d}=\frac{m_{p}}{\left(\sqrt{2} m_{d}\right)} \lim _{Q \rightarrow 0} \frac{1}{Q}\left[\mathcal{J}_{1,0}^{x}-\mathcal{J}_{0,1}^{x}\right]
$$

while the deuteron quadrupole moment is

$$
Q_{d}=\frac{\sqrt{2}}{m_{d}} \lim _{Q \rightarrow 0} \frac{1}{Q^{2}}\left[\mathcal{J}_{0,0}^{+}-\mathcal{J}_{1,1}^{+}\right] .
$$

We stress that, as was shown in [1], using the free current in the frame where $q^{+}=0$, in the limit $Q^{2} \rightarrow 0$ the angular condition is satisfied at the first order in $Q$, but it is violated at the second order. Therefore the angular condition is not a problem for the calculation of $\mu_{d}$, while the quadrupole moment is not uniquely determined within that approach.

From Eqs. (17,21) it is straightforward to obtain the expressions for the elastic structure functions $A\left(Q^{2}\right), B\left(Q^{2}\right)$ and for the tensor polarization $T_{20}\left(Q^{2}\right)$ in terms of the matrix elements of the free current $\mathcal{J}_{S_{z}, S_{z}}^{+}$and $\mathcal{J}_{S_{z}^{\prime}, S_{z}}^{x}$ :

$$
\begin{aligned}
& A\left(Q^{2}\right)=\frac{\zeta^{2}}{3}\left[\left(\mathcal{J}_{0,0}^{+}\right)^{2}+2\left(\mathcal{J}_{1,1}^{+}\right)^{2}+\left(\mathcal{J}_{1,0}^{x}-\mathcal{J}_{0,1}^{x}\right)^{2} / 2\right] \\
& B\left(Q^{2}\right)=\frac{1}{6 m_{d}^{2}}\left(\mathcal{J}_{1,0}^{x}-\mathcal{J}_{0,1}^{x}\right)^{2} \\
& T_{20}\left(Q^{2}\right)=-\zeta^{2} \frac{\sqrt{2}}{3} \frac{\left[\left(\mathcal{J}_{0,0}^{+}\right)^{2}-\left(\mathcal{J}_{1,1}^{+}\right)^{2}+f\left(\mathcal{J}_{1,0}^{x}-\mathcal{J}_{0,1}^{x}\right)^{2} / 4\right]}{A+B \tan ^{2}(\theta / 2)}
\end{aligned}
$$

\section{Deuteron front-form wave function and matrix ele- ments of the current operator}

We consider the deuteron as a system of two different, interacting particles with the same mass, $m=\left(m_{p}+m_{n}\right) / 2\left(m_{n}\right.$ is the neutron mass $)$, and spin $1 / 2$. For a system of $N$ particles with four-momenta $p_{i}(i=1,2, \ldots, N)$, FFHD internal variables $\boldsymbol{k}_{1}, \ldots, \boldsymbol{k}_{N}$ can be defined, such that $\sum_{i=1}^{N} \boldsymbol{k}_{i}=0$. The intrinsic three-momentum $\boldsymbol{k}_{i}$ is the spatial part of the four-vector

$$
k_{i}=L[\beta(G)]^{-1} p_{i}
$$

where $G=P_{0} / M_{0}$ is the four-velocity, and $P_{0}=p_{1}+\ldots+p_{N}$ the total four-momentum of a system of free particles, with $M_{0}=\left|P_{0}\right| \equiv\left|P_{0}^{2}\right|^{1 / 2}$. The matrix $\beta(G) \in \operatorname{SL}(2, \mathrm{C})$ (see Appendix B) represents a front-form boost. The action of the boost $L[\beta(G)]^{-1}$ is such that $P_{0}^{\prime}=L[\beta(G)]^{-1} P_{0} \equiv\left[\boldsymbol{P}_{0 \perp}^{\prime}=0, P_{0}^{\prime+}=M_{0}, P_{0}^{\prime-}=M_{0}\right]$.

Then the wave function for the deuteron internal state $\left|m_{d} 1 S_{z}\right\rangle \equiv\left|\chi_{1, S_{z}}\right\rangle$ can be written as follows 30

$$
\chi_{1, S_{z}}\left(\vec{k}_{\perp}, \xi, \sigma_{1}, \sigma_{2}\right)=\left\langle\vec{k}, \sigma_{1}, \sigma_{2} \mid \chi_{1, S_{z}}\right\rangle=\left\langle\vec{k}, \sigma_{1}, \sigma_{2}\left|R^{-1}\right| \Psi_{d}\right\rangle \omega(k)^{1 / 2}
$$


where $\xi=p_{1}^{+} / P^{+}$, and $\vec{k}_{\perp}=\vec{p}_{1 \perp}-\xi \vec{P}_{\perp}$. The internal three-momentum is $\vec{k}=\left(\vec{k}_{\perp}, k_{z}\right)$, where $k_{z}=(2 \xi-1) \omega(k), \omega(k)=\left(m^{2}+\vec{k}^{2}\right)^{1 / 2}$, and $k=|\vec{k}|$. It can be easily shown that $M_{0}=2 \omega(k)$. The normalization of $\left\langle\vec{k}, \sigma_{1}, \sigma_{2} \mid \chi_{1, S_{z}}\right\rangle$ is such that

$$
\sum_{\sigma_{1}, \sigma_{2}} \int\left|\left\langle\vec{k}, \sigma_{1}, \sigma_{2} \mid \chi_{1, S_{z}}\right\rangle\right|^{2} \frac{d \vec{k}}{(2 \pi)^{3} \omega(k)}=1
$$

The matrix $R$ is given by

$$
R=v\left(\vec{k}, \vec{s}_{1}\right) v\left(-\vec{k}, \vec{s}_{2}\right)
$$

where $v(\vec{k}, \vec{s})$ is the Melosh matrix 31, 15

$$
v(\vec{k}, \vec{s})=\frac{\omega(k)+m+k_{z}+\imath\left(\hat{\sigma}_{x} k_{y}-\hat{\sigma}_{y} k_{x}\right)}{\left[2(\omega(k)+m)\left(\omega(k)+k_{z}\right)\right]^{1 / 2}},
$$

while $\vec{s}_{1}$, and $\vec{s}_{2}$ are the usual nucleon spin operators, $\sigma_{1}$ and $\sigma_{2}$ the eigenvalues of $s_{1 z}$ and $s_{2 z}$, respectively, and $\hat{\sigma}_{i}$ the Pauli matrix operators. The generalized Melosh matrix can also be written as

$$
v(-\boldsymbol{k}, \boldsymbol{s})=\exp \left(\frac{\imath}{2} \varphi \boldsymbol{n} \hat{\boldsymbol{\sigma}}\right)
$$

with $\boldsymbol{n}=\left(\boldsymbol{e}_{z} \wedge \boldsymbol{k}\right) / k_{\perp}$, by defining the angle $\varphi$

$$
\varphi=2 \arctan \frac{k_{\perp}}{\omega(k)+m-k_{z}},
$$

The angle $\varphi$ will be used in the Appendix for the calculation of the deuteron quadrupole moment.

The wave function for the deuteron internal state obeys the mass equation

$$
M^{2} \chi_{1, S_{z}}\left(\vec{k}_{\perp}, \xi, \sigma_{1}, \sigma_{2}\right)=m_{d}^{2} \chi_{1, S_{z}}\left(\vec{k}_{\perp}, \xi, \sigma_{1}, \sigma_{2}\right)
$$

while the wave function $\Psi_{d}$ in Eq. (26) is the usual solution of the "nonrelativistic" Schroedinger equation. Indeed, if in the front-form dynamics the mass operator $\tilde{M}$ for the function $\Psi_{d}$ is defined by $\tilde{M}^{2}=R M^{2} R^{-1}=M_{0}^{2}+V$ with $V$ the interaction operator, then the mass equation $\tilde{M}^{2} \Psi_{d}=m_{d}^{2} \Psi_{d}$ has the same form as the "nonrelativistic" Schroedinger equation in momentum representation [32, 15]:

$$
\left(\frac{\vec{k}^{2}}{m}+\mathcal{V}\right) \Psi_{d}\left(\vec{k}, \sigma_{1}, \sigma_{2}\right)=E_{d} \Psi_{d}\left(\vec{k}, \sigma_{1}, \sigma_{2}\right)
$$

where

$$
\mathcal{V}=V / 4 m, \quad E_{d}=\left(m_{d}^{2}-4 m^{2}\right) / 4 m=\epsilon_{d}+\epsilon_{d}^{2} /(4 m)
$$

with $m_{d}=2 m+\epsilon_{d}$. Therefore the eigenvalue $E_{d}$ of Eq. (33) can be identified with the deuteron energy $\epsilon_{d}$, if the small quantity $\epsilon_{d}^{2} /(4 m)$ is disregarded. It has to be noted that, 
in the case of the $N-N$ interactions of the Nijmegen group [36], $E_{d}$ is directly linked through Eq. (34) to the deuteron energy $\epsilon_{d}$ used in their fits. For the continuous part of the two-nucleon spectrum the mass equation is identical to the "nonrelativistic" Schroedinger equation in momentum representation [16]. Therefore the operator $\mathcal{V}$ has to satisfy the same constraints of the potential as in nonrelativistic quantum mechanics and can be chosen to have any of the forms usually employed for the $N-N$ interaction in nonrelativistic nuclear physics.

Since the wave function $\Psi_{d}$ is an eigenstate of the standard nonrelativistic spin operator [9, 16, 17]

$$
\vec{S}_{n r}=\vec{l}(\vec{k})+\vec{s}_{1}+\vec{s}_{2}
$$

where $\vec{l}(\vec{k})$ is the usual orbital angular momentum, the Clebsh-Gordan coupling coefficients can be used. Then the internal deuteron wave function $\chi_{1, S_{z}}\left(\vec{k}, \sigma_{1}, \sigma_{2}\right)$ with polarization vector $\vec{e}_{S_{z}}$ (see Appendix A), is given by (cf. [9])

$$
\begin{aligned}
& \left\langle\vec{k}, \sigma_{1}, \sigma_{2} \mid \chi_{1, S_{z}}\right\rangle=(2 \pi)^{3 / 2} \sqrt{\omega(k) / 2} \sum_{\sigma_{1}^{\prime} \sigma_{2}^{\prime}}\left[v\left(\vec{k}, \vec{s}_{1}\right)^{-1}\right]_{\sigma_{1}, \sigma_{1}^{\prime}}\left[v\left(-\vec{k}, \vec{s}_{2}\right)^{-1}\right]_{\sigma_{2}, \sigma_{2}^{\prime}} \\
& {\left[\varphi_{0}(k) \delta_{i j}-\frac{1}{\sqrt{2}}\left(\delta_{i j}-\frac{3 k_{i} k_{j}}{k^{2}}\right) \varphi_{2}(k)\right]\left[\hat{\sigma}_{i} \hat{\sigma}_{y}\right]_{\sigma_{1}^{\prime}, \sigma_{2}^{\prime}}\left(\vec{e}_{S_{z}}\right)_{j}=} \\
& \left.2 \sqrt{\pi^{3} \omega(k)}\left(\vec{e}_{S_{z}}\right)_{j}\left[\chi_{0}(k) \delta_{i j}+\frac{3 k_{i} k_{j}}{\sqrt{2} k^{2}}\right) \varphi_{2}(k)\right]\left[v\left(\vec{k}, \vec{s}_{1}\right)^{-1} \hat{\sigma}_{i} \hat{\sigma}_{y} v\left(-\vec{k}, \vec{s}_{2}\right)^{*}\right]_{\sigma_{1}, \sigma_{2}}
\end{aligned}
$$

where a sum over the repeated indices $i, j=1,2,3$ is assumed and $\chi_{0}(k)=\varphi_{0}(k)-$ $(1 / \sqrt{2}) \varphi_{2}(k)$. The wave functions $\varphi_{0}(k)$ and $\varphi_{2}(k)$ coincide with the nonrelativistic $S$ and $D$ waves in momentum representation [32]. The normalization of $\varphi_{0}(k)$ and $\varphi_{2}(k)$ is such that $\int\left[\varphi_{0}(k)^{2}+\varphi_{2}(k)^{2}\right] d \vec{k}=1$. For the calculation of the matrix elements of the current it will be useful to put the internal deuteron wavefunction in a more compact form

$$
\chi_{1, S_{z}}\left(\vec{k}, \sigma_{1}, \sigma_{2}\right)=2 \sqrt{\pi^{3} \omega(k)}\left(\vec{e}_{S_{z}}\right)_{j} F_{i j}(\boldsymbol{k})\left[C_{i}(\boldsymbol{k})+\imath \hat{\boldsymbol{\sigma}} \cdot \boldsymbol{D}_{i}(\boldsymbol{k})\right]_{\sigma_{1}, \sigma_{2}}
$$

where

$$
F_{i j}(\boldsymbol{k})=\left[\chi_{0}(k) \delta_{i j}+\frac{3 k_{i} k_{j}}{\sqrt{2} k^{2}} \varphi_{2}(k)\right]
$$

and

$$
C_{i}(\boldsymbol{k})+\imath \hat{\boldsymbol{\sigma}} \cdot \boldsymbol{D}_{i}(\boldsymbol{k})=v\left(\boldsymbol{k}, \vec{s}_{1}\right)^{-1} \hat{\sigma}_{i} \hat{\sigma}_{y}\left[v\left(-\boldsymbol{k}, \vec{s}_{2}\right)\right]^{*}, \quad i=1,2,3
$$

In this paper the matrices $C_{i}(\boldsymbol{k})+\imath \hat{\boldsymbol{\sigma}} \cdot \boldsymbol{D}_{i}(\boldsymbol{k})$ will be called "generalized Melosh matrices for the deuteron wave function". Explicit expressions for the real quantities $C_{i}(\boldsymbol{k}), \boldsymbol{D}_{i}(\boldsymbol{k})$ can be found in Appendix C. 
The matrix elements $\mathcal{J}_{S_{z}^{\prime}, S_{z}}^{\mu}$ can be easily calculated, by using the action of the free current on a two-body state $\left|\vec{P}_{\perp}, P^{+}\right\rangle\left|\chi_{S, S_{z}}\right\rangle$ [30]:

$$
\begin{aligned}
& \left\langle p_{1}^{\prime}, p_{2}^{\prime} ; \sigma_{1}^{\prime}, \sigma_{2}^{\prime}\left|J_{\text {free }}^{\mu}(0)\right| \vec{P}_{\perp}, P^{+}\right\rangle\left|\chi_{S, S_{z}}\right\rangle=\sum_{\sigma_{1}} \bar{w}\left(p_{1}^{\prime}, \sigma_{1}^{\prime}\right) \cdot \\
& \left\{2 m\left[f_{e}^{i s}\left(\left(p_{1}^{\prime}-p_{1}\right)^{2}\right)-f_{m}^{i s}\left(\left(p_{1}^{\prime}-p_{1}\right)^{2}\right)\right] \frac{\left(p_{1}+p_{1}^{\prime}\right)^{\mu}}{\left(p_{1}+p_{1}^{\prime}\right)^{2}}+f_{m}^{i s}\left(\left(p_{1}^{\prime}-p_{1}\right)^{2}\right) \gamma^{\mu}\right\} . \\
& w\left(p_{1}, \sigma_{1}\right)\left\langle\vec{k}, \sigma_{1}, \sigma_{2}^{\prime} \mid \chi_{S, S_{z}}\right\rangle \frac{1}{\xi}
\end{aligned}
$$

where, in our case,

$$
J_{\text {free }}^{\mu}(0)=J_{p}^{\mu}(0)+J_{n}^{\mu}(0) .
$$

In Eq. (40) $w(p, \sigma)$ is the front-form Dirac spinor [30] (see Appendix B), while $f_{e}^{i s}=f_{e}^{p}+f_{e}^{n}$ and $f_{m}^{i s}=f_{m}^{p}+f_{m}^{n}$ are the isoscalar electric and magnetic Sachs form factors of the nucleon.

An explicit calculation, with the help of the matrix elements of the $\gamma$ matrices between front-form Dirac spinors reported in Appendix B, shows that, as a consequence of Eqs. (40,41),

$$
\begin{aligned}
& \left\langle\chi_{1, S_{z}}\left|\mathcal{J}^{+}\left(K \vec{e}_{z}, m_{d}, m_{d}\right)\right| \chi_{1, S_{z}}\right\rangle=\left\langle\chi_{1, S_{z}}\left|\left\langle 0, K^{\prime}+\left|J_{\text {free }}^{+}(0)\right| 0, K^{+}\right\rangle\right| \chi_{1, S_{z}}\right\rangle= \\
& \sqrt{2} m_{d} \sum_{\sigma_{1}, \sigma_{1}^{\prime} \sigma_{2}} \int \chi_{1, S_{z}}\left(\vec{k}^{\prime}, \sigma_{1}^{\prime}, \sigma_{2}\right)^{*}\left\{\frac{a m\left(f_{e}^{i s}-f_{m}^{i s}\right)\left[a m+\imath b(\hat{\sigma} k)_{\perp}\right]}{a^{2} m^{2}+b^{2} \vec{k}_{\perp}^{2}}+f_{m}^{i s}\right\}_{\sigma_{1}^{\prime} \sigma_{1}} . \\
& \chi_{1, S_{z}}\left(\vec{k}, \sigma_{1}, \sigma_{2}\right)\left(\xi \xi^{\prime}\right)^{1 / 2} \frac{d \vec{k}^{\prime}}{(2 \pi)^{3} \omega\left(k^{\prime}\right) \xi} \\
& \left\langle\chi_{1, S_{z}^{\prime}}\left|\mathcal{J}_{x}\left(K \vec{e}_{z}, m_{d}, m_{d}\right)\right| \chi_{1, S_{z}}\right\rangle=\left\langle\chi_{1, S_{z}^{\prime}}\left|\left\langle 0, K^{\prime}+\left|J_{f r e e}^{x}(0)\right| 0, K^{+}\right\rangle\right| \chi_{1, S_{z}}\right\rangle= \\
& \sum_{\sigma_{1}, \sigma_{1}^{\prime} \sigma_{2}} \int \chi_{1, S_{z}^{\prime}}\left(\vec{k}^{\prime}, \sigma_{1}^{\prime}, \sigma_{2}\right)^{*}\left\{\frac{4 m k_{x}\left(f_{e}^{i s}-f_{m}^{i s}\right)\left[a m+\imath b(\hat{\sigma} k)_{\perp}\right]}{a^{2} m^{2}+b^{2} \vec{k}_{\perp}^{2}}+f_{m}^{i s}\left[a k_{x}+\imath b\left(m \hat{\sigma}_{y}+k_{y} \hat{\sigma}_{z}\right)\right]\right\} \\
& \chi_{1, S_{z}}\left(\vec{k}, \sigma_{1}, \sigma_{2}\right) \frac{d \vec{k}^{\prime}}{(2 \pi)^{3} \omega\left(k^{\prime}\right) \xi}
\end{aligned}
$$

where

$$
a=\left[\frac{K^{\prime}+\xi^{\prime}}{K^{+} \xi}\right]^{1 / 2}+\left[\frac{K^{+} \xi}{K^{\prime}+\xi^{\prime}}\right]^{1 / 2}, \quad b=\left[\frac{K^{\prime}+\xi^{\prime}}{K^{+} \xi}\right]^{1 / 2}-\left[\frac{K^{+} \xi}{K^{\prime}+\xi^{\prime}}\right]^{1 / 2}
$$

and the form factors $f_{e}^{i s}$ and $f_{m}^{i s}$ are functions of $\left(p_{1}^{\prime}-p_{1}\right)^{2}$. In our Breit reference frame, where $\vec{K}_{\perp}=0$ and $\vec{q}_{\perp}=0$, the relations between the internal $\left(\vec{k}_{\perp}, k_{z}\right)$ and individual nucleon 
variables, in the initial, $\chi_{1, S_{z}}\left(\vec{k}, \sigma_{1}, \sigma_{2}\right)$, and final, $\chi_{1, S_{z}^{\prime}}\left(\vec{k}^{\prime}, \sigma_{1}^{\prime}, \sigma_{2}^{\prime}\right)$, wave functions are given by

$$
\begin{aligned}
& \vec{p}_{1 \perp}=\vec{p}_{1 \perp}^{\prime}=\vec{k}_{\perp}=\vec{k}_{\perp}^{\prime}, \quad p_{1}^{+}=\xi K^{+}, \quad k_{z}=\omega(k)(2 \xi-1), \quad k_{z}^{\prime}=\omega\left(k^{\prime}\right)\left(2 \xi^{\prime}-1\right), \\
& \xi^{\prime}=\frac{p_{1}^{\prime+}}{K^{\prime}+}=1+(\xi-1) \frac{K^{+}}{K^{\prime}}=\frac{\xi\left[\sqrt{m_{d}^{2}+K^{2}}-K\right]+2 K}{\sqrt{m_{d}^{2}+K^{2}}+K}=\frac{\xi\left[\sqrt{1+\kappa^{2}}-\kappa\right]+2 \kappa}{\sqrt{1+\kappa^{2}}+\kappa}
\end{aligned}
$$

with $\kappa=K / m_{d}$. It is important to note that nucleon form factors cannot be factorized out in the current matrix elements, since from Eq. (45) one has

$$
\left(p_{1}^{\prime}-p_{1}\right)^{2}=-4 \tau\left(m^{2}+\vec{k}_{\perp}^{2}\right) /\left(\xi \xi^{\prime}\right) \neq-Q^{2} .
$$

By using the expression (36) of the deuteron wave function, a direct calculation shows that

$$
\begin{aligned}
& \left\langle\chi_{1, S_{z}}\left|\mathcal{J}^{+}\left(K \vec{e}_{z}, m_{d}, m_{d}\right)\right| \chi_{1, S_{z}}\right\rangle=\sqrt{2} m_{d}\left(\vec{e}_{S_{z}}\right)_{j^{\prime}}^{*}\left(\vec{e}_{S_{z}}\right)_{j} \int\left[\frac{\omega(k) \xi^{\prime}}{\omega\left(k^{\prime}\right) \xi}\right]^{1 / 2} . \\
& {\left[\chi_{0}\left(k^{\prime}\right) \delta_{i^{\prime} j^{\prime}}+\frac{3 k_{i^{\prime}}^{\prime} k_{j^{\prime}}^{\prime}}{\sqrt{2}{k^{\prime}}^{2}} \varphi_{2}\left(k^{\prime}\right)\right]\left[\chi_{0}(k) \delta_{i j}+\frac{3 k_{i} k_{j}}{\sqrt{2} k^{2}} \varphi_{2}(k)\right] .} \\
& \frac{1}{2} \operatorname{Tr}\left\{\left[v\left(-\boldsymbol{k}^{\prime}, \vec{s}_{2}\right)\right]^{T} \hat{\sigma}_{y} \hat{\sigma}_{i^{\prime}} v\left(\boldsymbol{k}^{\prime}, \vec{s}_{1}\right)\left[\frac{a m\left(f_{e}^{i s}-f_{m}^{i s}\right)\left[a m+\imath b(\hat{\sigma} k)_{\perp}\right]}{a^{2} m^{2}+b^{2} k_{\perp}^{2}}+f_{m}^{i s}\right]\right. \text {. } \\
& \left.v\left(\boldsymbol{k}, \vec{s}_{1}\right)^{-1} \hat{\sigma}_{i} \hat{\sigma}_{y}\left[v\left(-\boldsymbol{k}, \vec{s}_{2}\right)\right]^{*}\right\} d \boldsymbol{k}^{\prime} \\
& \left\langle\chi_{1, S_{z}^{\prime}}\left|\mathcal{J}_{x}\left(K \vec{e}_{z}, m_{d}, m_{d}\right)\right| \chi_{1, S_{z}}\right\rangle=\left(\vec{e}_{S_{z}^{\prime}}\right)_{j^{\prime}}^{*}\left(\vec{e}_{S_{z}}\right)_{j} \int\left[\frac{\omega(k)}{\omega\left(k^{\prime}\right)}\right]^{1 / 2} . \\
& {\left[\chi_{0}\left(k^{\prime}\right) \delta_{i^{\prime} j^{\prime}}+\frac{3 k_{i^{\prime}}^{\prime} k_{j^{\prime}}^{\prime}}{\sqrt{2}{k^{\prime}}^{2}} \varphi_{2}\left(k^{\prime}\right)\right]\left[\chi_{0}(k) \delta_{i j}+\frac{3 k_{i} k_{j}}{\sqrt{2} k^{2}} \varphi_{2}(k)\right] \text {. }} \\
& \frac{1}{2} \operatorname{Tr}\left\{[ v ( - \boldsymbol { k } ^ { \prime } , \vec { s } _ { 2 } ) ] ^ { T } \hat { \sigma } _ { y } \hat { \sigma } _ { i ^ { \prime } } v ( \boldsymbol { k } ^ { \prime } , \vec { s } _ { 1 } ) \left\{\frac{4 m k_{x}\left(f_{e}^{i s}-f_{m}^{i s}\right)\left[a m+\imath b(\hat{\sigma} k)_{\perp}\right]}{a^{2} m^{2}+b^{2} k_{\perp}^{2}}+\right.\right. \\
& \left.\left.f_{m}^{i s}\left[a k_{x}+\imath b\left(m \hat{\sigma}_{y}+k_{y} \hat{\sigma}_{z}\right)\right]\right\} v\left(\boldsymbol{k}, \vec{s}_{1}\right)^{-1} \hat{\sigma}_{i} \hat{\sigma}_{y}\left[v\left(-\boldsymbol{k}, \vec{s}_{2}\right)\right]^{*}\right\} \frac{d \boldsymbol{k}^{\prime}}{\xi}
\end{aligned}
$$

where the superscript $T$ on a matrix indicates the transposition of the matrix and a sum over the repeated indices $i, j, i^{\prime}, j^{\prime}$ is understood. By means of the matrices $C_{i}(\boldsymbol{k})+\imath \hat{\boldsymbol{\sigma}} \cdot \boldsymbol{D}_{i}(\boldsymbol{k})$, equations (47), (48) can be rewritten as follows :

$$
\begin{aligned}
& \left\langle\chi_{1, S_{z}}\left|\mathcal{J}^{+}\left(K \vec{e}_{z}, m_{d}, m_{d}\right)\right| \chi_{1, S_{z}}\right\rangle=\sqrt{2} m_{d}\left(\vec{e}_{S_{z}}\right)_{j^{\prime}}^{*}\left(\vec{e}_{S_{z}}\right)_{j} \int\left[\frac{\omega(k) \xi^{\prime}}{\omega\left(k^{\prime}\right) \xi}\right]^{1 / 2} F_{i^{\prime} j^{\prime}}\left(\boldsymbol{k}^{\prime}\right) F_{i j}(\boldsymbol{k}) . \\
& \frac{1}{2} \operatorname{Tr}\left\{\left[C_{i^{\prime}}\left(\boldsymbol{k}^{\prime}\right)-\imath \hat{\boldsymbol{\sigma}} \cdot \boldsymbol{D}_{i^{\prime}}\left(\boldsymbol{k}^{\prime}\right)\right]\left[A^{+}+\imath \hat{\boldsymbol{\sigma}} \cdot \boldsymbol{B}^{+}\right]\left[C_{i}(\boldsymbol{k})+\imath \hat{\boldsymbol{\sigma}} \cdot \boldsymbol{D}_{i}(\boldsymbol{k})\right]\right\} d \boldsymbol{k}^{\prime}
\end{aligned}
$$




$$
\begin{aligned}
& \left\langle\chi_{1, S_{z}^{\prime}}\left|\mathcal{J}_{x}\left(K \vec{e}_{z}, m_{d}, m_{d}\right)\right| \chi_{1, S_{z}}\right\rangle=\left(\vec{e}_{S_{z}^{\prime}}\right)_{j^{\prime}}^{*}\left(\vec{e}_{S_{z}}\right)_{j} \int\left[\frac{\omega(k)}{\omega\left(k^{\prime}\right)}\right]^{1 / 2} F_{i^{\prime} j^{\prime}}\left(\boldsymbol{k}^{\prime}\right) F_{i j}(\boldsymbol{k}) \cdot \\
& \frac{1}{2} \operatorname{Tr}\left\{\left[C_{i^{\prime}}\left(\boldsymbol{k}^{\prime}\right)-\imath \hat{\boldsymbol{\sigma}} \cdot \boldsymbol{D}_{i^{\prime}}\left(\boldsymbol{k}^{\prime}\right)\right]\left[A_{x}+\imath \hat{\boldsymbol{\sigma}} \cdot \boldsymbol{B}_{x}\right]\left[C_{i}(\boldsymbol{k})+\imath \hat{\boldsymbol{\sigma}} \cdot \boldsymbol{D}_{i}(\boldsymbol{k})\right]\right\} \frac{d \boldsymbol{k}^{\prime}}{\xi}
\end{aligned}
$$

where

$$
A^{+}+\imath \hat{\boldsymbol{\sigma}} \cdot \boldsymbol{B}^{+}=\frac{a m\left(f_{e}^{i s}-f_{m}^{i s}\right)\left[a m+\imath b(\hat{\sigma} k)_{\perp}\right]}{a^{2} m^{2}+b^{2} k_{\perp}^{2}}+f_{m}^{i s}
$$

and

$$
A_{x}+\imath \hat{\boldsymbol{\sigma}} \cdot \boldsymbol{B}_{x}=\frac{4 m k_{x}\left(f_{e}^{i s}-f_{m}^{i s}\right)\left[a m+\imath b(\hat{\sigma} k)_{\perp}\right]}{a^{2} m^{2}+b^{2} k_{\perp}^{2}}+f_{m}^{i s}\left[a k_{x}+\imath b\left(m \hat{\sigma}_{y}+k_{y} \hat{\sigma}_{z}\right)\right] .
$$

It is straightforward to see that $A_{x}$ is proportional to the quantity $a$, while $\boldsymbol{B}^{+}$and $\boldsymbol{B}_{x}$ are proportional to $b$, defined in Eq. (44). All the quantities $A^{+}, \boldsymbol{B}^{+}, A_{x}, \boldsymbol{B}_{x}, C_{i}(\boldsymbol{k}), \boldsymbol{D}_{i}(\boldsymbol{k})$ are real.

By an explicit calculation of the traces in Eqs. (49), (50) one has

$$
\begin{aligned}
& \left\langle\chi_{1, S_{z}}\left|\mathcal{J}^{+}\left(K \vec{e}_{z}, m_{d}, m_{d}\right)\right| \chi_{1, S_{z}}\right\rangle=\sqrt{2} m_{d}\left(\vec{e}_{S_{z}}\right)_{j^{\prime}}^{*}\left(\vec{e}_{S_{z}}\right)_{j} \int\left[\frac{\omega(k) \xi^{\prime}}{\omega\left(k^{\prime}\right) \xi}\right]^{1 / 2} F_{i^{\prime} j^{\prime}}\left(\boldsymbol{k}^{\prime}\right) F_{i j}(\boldsymbol{k}) \cdot \\
& \left\{A^{+}\left[C_{i^{\prime}}\left(\boldsymbol{k}^{\prime}\right) C_{i}(\boldsymbol{k})+\boldsymbol{D}_{i^{\prime}}\left(\boldsymbol{k}^{\prime}\right) \cdot \boldsymbol{D}_{i}(\boldsymbol{k})\right]-\right. \\
& \left.\boldsymbol{B}^{+} \cdot\left[C_{i^{\prime}}\left(\boldsymbol{k}^{\prime}\right) \boldsymbol{D}_{i}(\boldsymbol{k})-\boldsymbol{D}_{i^{\prime}}\left(\boldsymbol{k}^{\prime}\right) C_{i}(\boldsymbol{k})-\boldsymbol{D}_{i^{\prime}}\left(\boldsymbol{k}^{\prime}\right) \wedge \boldsymbol{D}_{i}(\boldsymbol{k})\right]\right\} d \boldsymbol{k}^{\prime} \\
& \left\langle\chi_{1, S_{z}^{\prime}}\left|\mathcal{J}_{x}\left(K \vec{e}_{z}, m_{d}, m_{d}\right)\right| \chi_{1, S_{z}}\right\rangle=\left(\vec{e}_{S_{z}^{\prime}}\right)_{j^{\prime}}^{*}\left(\vec{e}_{S_{z}}\right)_{j} \int\left[\frac{\omega(k)}{\omega\left(k^{\prime}\right)}\right]^{1 / 2} F_{i^{\prime} j^{\prime}}\left(\boldsymbol{k}^{\prime}\right) F_{i j}(\boldsymbol{k}) \cdot \\
& \left\{A_{x}\left[C_{i^{\prime}}\left(\boldsymbol{k}^{\prime}\right) C_{i}(\boldsymbol{k})+\boldsymbol{D}_{i^{\prime}}\left(\boldsymbol{k}^{\prime}\right) \cdot \boldsymbol{D}_{i}(\boldsymbol{k})\right]-\right. \\
& \left.\quad \boldsymbol{B}_{x} \cdot\left[C_{i^{\prime}}\left(\boldsymbol{k}^{\prime}\right) \boldsymbol{D}_{i}(\boldsymbol{k})-\boldsymbol{D}_{i^{\prime}}\left(\boldsymbol{k}^{\prime}\right) C_{i}(\boldsymbol{k})-\boldsymbol{D}_{i^{\prime}}\left(\boldsymbol{k}^{\prime}\right) \wedge \boldsymbol{D}_{i}(\boldsymbol{k})\right]\right\} \frac{d \boldsymbol{k}^{\prime}}{\xi}
\end{aligned}
$$

It has to be noted that the integrals in Eqs. (53, 54) are real. Therefore, since the matrix

elements $\mathcal{J}_{S_{z}, S_{z}}^{+}$and $\mathcal{J}_{S_{z}^{\prime}, S_{z}}^{x}$ are real (see the end of Sect. 2), only the real part of $\left(\vec{e}_{S_{z}^{\prime}}\right)_{j^{\prime}}^{*}\left(\vec{e}_{S_{z}}\right)_{j}$ can contribute to these matrix elements.

\section{Numerical results for the deuteron form factors}

\subsection{Deuteron magnetic and quadrupole moments}

The direct evaluation of magnetic and quadrupole moments through the limits of Eqs. (22,23) implies very delicate numerical problems and then a careful analitical reduction of these 
equation is needed. For the sake of completeness we report in Appendix D the explicit expressions that have actually been used. Magnetic and quadrupole moments have already been calculated in Ref. [13] for a variety of $N-N$ interactions. In this paper we recall our main results, which are summarized in Table I. In the table the values of the magnetic and quadrupole moments calculated with many $N-N$ interactions, already shown in Ref. [13], are reported together with the values obtained using the local Nijmegen 2 interaction, which was not considered in Ref. [13].

The standard non-relativistic results obtained with a one-body current crucially depend on the asymptotic normalization ratio $\eta=A_{D} / A_{S}$ of $D$ and $S$ wave functions and on the $D$-state percentage in the deuteron, $P_{D}$, but one cannot obtain at the same time the experimental values for both $\mu_{d}$ and $Q_{d}$. Using the free current within the FFHD in the $q^{+}=0$ reference frame, the relativistic correction (RC) turned out to be very small for $Q_{d}$, while for $\mu_{d}$ it could explain only part of the disagreement with the experimental value [9]. On the contrary, in our Poincaré covariant calculation [13 the RC's bring both $\mu_{d}$ and $Q_{d}$ closer to the experimental values, except for the charge-dependent Bonn interaction [37]. We wish to stress that our current operator and the one used in Ref. 99 are different, since both of them are obtained from the free one, but in different reference frames, related by an interaction dependent rotation. As was already observed for the nonrelativistic calculations of $Q_{d}$ [38, 39], we have shown in Ref. [13] that a remarkable linear behaviour against the asymptotic normalization ratio, $\eta$, holds for both the deuteron moments calculated within our approach (the values of $\mu_{d}$ and $Q_{d}$ corresponding to the Nijmegen 2 interaction obey precisely the same trend as the other interactions). The values of $\mu_{d}$ and $Q_{d}$, suggested by this linear behaviour in correspondence of the experimental value of $\eta\left(\eta^{e x p}=0.0256(4)\right.$ 40]) differ from the experimental ones $\left(\mu_{d}=0.857406(1)\right.$ [41] and $Q_{d}=0.2859(3)$ [38] ) only by $0.5 \%$ and $2 \%$, respectively, i.e. much less than for the non-relativistic results. The RC to $\mu_{d}$ is rather large and the total result becomes slightly greater than $\mu_{d}^{e x p}$, while the nonrelativistic one is smaller. This shows that, within our framework, even the sign of explicit contributions of two-body currents is different from the one needed in the non-relativistic case. In conclusion, it appears that, within our approach, the total contribution of two-body currents (from meson-exchange, Z-graphs, etc.) and isobar configurations has to be relatively small at $Q^{2}=0$.

\subsection{Deuteron form factors and $N-N$ interactions}

Let us first compare in Figs. 1 and 2 our relativistic results for $A\left(Q^{2}\right), B\left(Q^{2}\right)$ and $T_{20}\left(Q^{2}\right)$, obtained using the $R S C$ interaction [33] and the Gari-Krümpelmann nucleon form factors [42], with the corresponding nonrelativistic results. Following Lomon [24], the latter ones have been obtained by using the exact relativistic relations between the deuteron form factors and the current matrix elements, within the Breit reference frame where the momentum transfer is directed along the $z$ axis [43], but with nonrelativistic expressions for the matrix elements evaluated in impulse approximation [24].

In order to have a closer insight to the form factor behaviour, in addition to the 
usual plots for $A\left(Q^{2}\right)$ and $B\left(Q^{2}\right)$ in a logarithmic scale, shown in Fig. 1, we report in Fig. 2(a) the quantity $A\left(Q^{2}\right)$ divided by the factor $\left(G_{D}^{2} \cdot F\right)$, with $G_{D}=\left(1+Q^{2} / 0.71\right)^{-2}$ and $F=\left(1+Q^{2} / 0.1\right)^{-2.5}$, in a linear scale, and in Fig. 2(b) the quantity $\Gamma_{M}\left(Q^{2}\right)=$ $\left[G_{M}\left(Q^{2}\right) m_{p} /\left(\mu_{d} m_{d}\right)\right]^{2}$ divided by the factor $\left(G_{D}^{2} \cdot F_{1}\right)$, with $F_{1}=\left(1+Q^{2} / 0.1\right)^{-3}$. As it is clear from Figs. 1 and 2, the differences between relativistic and nonrelativistic results are a few percent for $Q^{2} \leq 0.1(\mathrm{GeV} / \mathrm{c})^{2}$, while become large as $Q^{2}$ increases. For $A\left(Q^{2}\right)$ the differences are larger than $20 \%$ already at $Q^{2} \geq 0.2(\mathrm{GeV} / \mathrm{c})^{2}$ and are of orders of magnitude for $Q^{2} \geq$ $2(\mathrm{GeV} / \mathrm{c})^{2}$. For $B\left(Q^{2}\right)$ the relativistic and nonrelativistic results differ by $50-100 \%$ for $Q^{2} \geq 0.3(\mathrm{GeV} / \mathrm{c})^{2}$, while for $T_{20}\left(Q^{2}\right)$ they considerably differ for $Q^{2} \geq 0.5(\mathrm{GeV} / \mathrm{c})^{2}$. In Figs. 1, 2 we have also reported by dashed lines the results obtained by keeping fixed the argument of the nucleon form factors in Eqs. (47,48). The effects of factorization become large for $A\left(Q^{2}\right)$ and $B\left(Q^{2}\right)$ at $Q^{2} \geq 1(G e V / c)^{2}$, while for $T_{20}\left(Q^{2}\right)$ already at $Q^{2} \geq 0.5(\mathrm{GeV} / \mathrm{c})^{2}$. From Fig. 1 it appears that the nonrelativistic approach is able to give an overall description of the data for $A\left(Q^{2}\right), B\left(Q^{2}\right)$, and $T_{20}\left(Q^{2}\right)$. However, this description is not accurate, even at very low values of the momentum transfer, as one can see in Fig. 2 (a) and, furthermore, it strongly depends on the $N-N$ interaction and the nucleon form factor model. For instance, using the $C D-$ Bonn interaction [37] and the nucleon form factors by Hoehler et al. [60], for $A\left(Q^{2}\right)$ and $T_{20}\left(Q^{2}\right)$ the agreement is completely lost at $Q^{2} \geq 0.4(\mathrm{GeV} / \mathrm{c})^{2}$.

A comparison of our results with the deuteron form factors obtained by using the same $N-N$ interactions and the same nucleon form factors, but within different relativistic approaches, for instance within the front-form calculation of Ref. [9], can also be interesting. Using the Paris interaction [35] and the form factors of Ref. [60], large differences have been found for $A\left(Q^{2}\right)$ at $Q^{2} \geq 2(G e V / c)^{2}$, which become of orders of magnitude at $Q^{2}=$ $6(\mathrm{GeV} / \mathrm{c})^{2}$ (see Ref. 27]). For $B\left(Q^{2}\right)$ we found a minimum around $Q^{2}=1.8(\mathrm{GeV} / \mathrm{c})^{2}$ instead of $Q^{2}=1.6(\mathrm{GeV} / \mathrm{c})^{2}$ as in Ref. [9], and for $T_{20}\left(Q^{2}\right)$ a zero at $Q^{2}=1.4(\mathrm{GeV} / \mathrm{c})^{2}$ instead of $1.2(\mathrm{GeV} / \mathrm{c})^{2}$.

The results obtained within our approach with different $N-N$ interactions are analyzed in Figs. 3 and 4, using the nucleon form factor model by Hoehler et al. [60]. We consider the old RSC interaction [33] and recent realistic interactions, able to describe the two-body data with a reduced $\chi^{2} \approx 1$. In particular we study the $A V 18$ interaction by the Argonne group [25], some interactions by the Nijmegen group (Nijmegen1, Nijmegen2, Nijmegen93, Reid93) [36], and the charge-dependent $C D-$ Bonn interaction by the Bonn group [37. The results for the Reid93 interaction are essentially equal to the results of the $A V 18$ interaction and are not reported in the figures.

The effects of different interactions are large for $A\left(Q^{2}\right)$ at $Q^{2} \geq 1(\mathrm{GeV} / \mathrm{c})^{2}$, while for $B\left(Q^{2}\right)$ and $T_{20}\left(Q^{2}\right)$ already at $Q^{2} \geq 0.5(\mathrm{GeV} / \mathrm{c})^{2}$. It can be noted that the $C D-B o n n$ interaction, which is characterized by a larger non-locality, yields larger differences with respect to the other interactions. At low values of $Q^{2}\left(Q^{2}<0.4(\mathrm{GeV} / \mathrm{c})^{2}\right)$, where the nucleon form factors are better known, a simultaneous description of the experimental data for $A\left(Q^{2}\right)$, $B\left(Q^{2}\right)$ and $T_{20}\left(Q^{2}\right)$ is achieved. The dependence on the nucleon-nucleon interaction in this region is minor, although not negligible (see, in particular, Fig. 4 (b)).

For the mentioned interactions and using the Gari-Krümpelmann nucleon form factors 
[42], we report in Fig. 5 (a) the value of $Q^{2}$ corresponding to the minimum of $B\left(Q^{2}\right)$ and in Fig. 5 (b) the value of $Q^{2}$ corresponding to the second zero of $T_{20}\left(Q^{2}\right)$ against the nonrelativistic S-state kinetic energy, $T_{S}$, in order to find a correlation between different effects of the $N-N$ interactions. For both quantities a distinct linear behaviour is clear: a lower value of $T_{S}$ yields a minimum for $B\left(Q^{2}\right)$ and a zero for $T_{20}\left(Q^{2}\right)$ at a larger momentum transfer. Analogous results can be obtained with different nucleon form factors, as the ones of Ref. 60. From Figs. 3(a), 4(a) it is clear that for $Q^{2} \geq 1(\mathrm{GeV} / \mathrm{c})^{2}$ a similar correlation holds for $A\left(Q^{2}\right)$, i.e. a lower value of $T_{S}$ yields a lower value of $A\left(Q^{2}\right)$. It has also to be noted that the $A V 18$ and Reid93 interactions, which give essentially the same results for $A\left(Q^{2}\right), B\left(Q^{2}\right)$ and $T_{20}\left(Q^{2}\right)$, have the same S-state kinetic energy.

Let us note that recent measurements of the $S-D$ mixing parameter, $\epsilon_{1}$, point to a stronger tensor force than the one exhibited by the interaction models we have analyzed [61]. In turn, a stronger tensor force is favoured by a high degree of locality, which yields significantly larger kinetic energies and, in particular, larger values of $T_{S}$ 62]. Then, by an extrapolation of the linear relations found above, one can argue that a $N-N$ interaction able to reproduce these recent measurements of $\epsilon_{1}$ could yield, on one hand, agreement between experimental and theoretical values for $T_{20}\left(Q^{2}\right)$ and, on the other one, a minimum for $B\left(Q^{2}\right)$ slightly lower than the value indicated by the available experimental data (around $Q^{2}=$ $1.6(\mathrm{GeV} / \mathrm{c})^{2}$ instead of $\left.Q^{2}=1.8(\mathrm{GeV} / \mathrm{c})^{2}\right)$. Therefore, if new, more precise experimental data for $B\left(Q^{2}\right)$ will show such a lower value for the position of the minimum, both $B\left(Q^{2}\right)$ and $T_{20}\left(Q^{2}\right)$ could be reproduced by a novel $N-N$ interaction, without a relevant role for explicit two-body currents.

\subsection{Deuteron form factors and nucleon electromagnetic form fac- tors}

In order to investigate the effects of the nucleon form factors on the deuteron form factors, we have displayed in Fig. 6 our results obtained with the Nijmegen 2 nucleon-nucleon interaction and corresponding to the nucleon form factor models of Refs. [42], [60], and [63]. For $A\left(Q^{2}\right)$ the differences between different models are very large at $Q^{2} \geq 0.5(\mathrm{GeV} / \mathrm{c})^{2}$, increase as $Q^{2}$ increases, and can be related to the sizeably different behaviour of $G_{E}^{n}\left(Q^{2}\right)$ for the various models. The influence of the nucleon form factor models is less marked in $B\left(Q^{2}\right)$, while, as already known [8], the tensor polarization is essentially independent of the nucleon form factors.

Therefore, the linear behaviour of the locations of the minimum of $B\left(Q^{2}\right)$ and the second zero of $T_{20}\left(Q^{2}\right)$ vs. $T_{S}$ is substantially independent of the form factor models, as well as the conjecture at the end of the previous paragraph. As far as $A\left(Q^{2}\right)$ is concerned, one could try to exploit the strong dependence of $A\left(Q^{2}\right)$ on $G_{E}^{n}\left(Q^{2}\right)$ to gain information on $G_{E}^{n}\left(Q^{2}\right)$ by a fit of the $A\left(Q^{2}\right)$ experimental data, following a procedure analogous to the one used, in a nonrelativistic context, by Platchkov et al. [45]. Obviously the results of this fit will be different for different interactions. Another possibility to be studied in our covariant framework is obviously the role of isobar configurations in the deuteron state (see, e.g. [7]) 
and of explicit two-body contributions in the e.m. current (see, e.g. [2]). As already noted [10], [30], these contributions have to be Poincaré covariant, and to satisfy Hermiticity and current conservation by themselves. We intend to perform such a fit and to study these contributions elsewhere.

\section{Conclusions}

In this paper the deuteron form factors $A\left(Q^{2}\right)$ and $B\left(Q^{2}\right)$, and the tensor polarization $T_{20}\left(Q^{2}\right)$ have been evaluated in the framework of front-form Hamiltonian dynamics, using a Poincaré covariant current operator, without any ambiguity. The current is built up from the free one in the Breit reference frame where $\vec{q}$ is along the $z$ axis and fulfills parity and time reversal covariance, as well as Hermiticity and current conservation.

Large differences have been found between the results of calculations performed within a nonrelativistic framework and within our Poincaré covariant approach. These differences become huge at high momentum transfer, as expected, but are relevant for accurate calculations even in the limit of zero momentum transfer, as is clear from our results for the deuteron magnetic and quadrupole moments [13]. Large differences have also been found with respect to a front-form approach which ensures Poincaré covariance by different definitions for different matrix elements of the current operator (9]). Our current operator, which was already shown to be able to describe the deuteron magnetic and quadrupole moments, is also able to simultaneously reproduce the three deuteron form factors at low momentum transfer, where the nucleon form factor are better known and the effects of different interactions are minor.

The effects on the deuteron form factors of different nucleon-nucleon interactions and different nucleon form factor models have been studied. The different nucleon form factor models strongly affect $A\left(Q^{2}\right)$, while the different interactions have large effects on $A, B$ and $T_{20}$. These effects are linked to the $S$-state kinetic energy in the deuteron, which, in turn, is related to the degree of non-locality of the interactions and to the strenght of the tensor force. A novel $N-N$ interaction with a strong tensor force, able to reproduce the recent measurements of $\epsilon_{1}$, would be helpful to describe the deuteron form factors and, in particular, to offer a solid ground for the study of the neutron charge form factor from the analysis of $A\left(Q^{2}\right)$. We stress the relevance of a well defined relativistic approach to gain reliable information on the nucleon-nucleon interaction and the nucleon form factors.

\section{Acknowledgements}

The authors wish to thank A. Kievsky for kindly providing the deuteron wavefunctions for RSC, and Av18 interactions and R. Machleidt for the CD-Bonn wavefunction. The wave functions for the Nijmegen interactions have been downloaded from: http://nn-online.sci.kun.n

. This work was partially supported by Ministero della Ricerca Scientifica e Tecnologica.

\section{Appendix}


In this Appendix we report for the sake of completeness the explicit expressions of some useful quantities needed for the calculation of the deuteron em form factors.

\section{A Polarization vectors}

The deuteron polarization four-vectors, $e_{S_{z}}$, in any reference frame can be obtained by a proper boost from the polarization vectors in the deuteron rest frame, $e_{S_{z}}(r f) \equiv\left(e_{r f}^{0}=\right.$ $\left.0, \vec{e}_{S_{z}}\right)$, with

$$
\vec{e}_{+1}=-\frac{1}{\sqrt{2}}(1, \imath, 0), \quad \vec{e}_{-1}=\frac{1}{\sqrt{2}}(1,-\imath, 0), \quad \vec{e}_{0}=(0,0,1)
$$

In our Breit frame, where $\vec{P}_{\perp}=\vec{P}_{\perp}^{\prime}=0$, the transverse deuteron polarization vectors, in both the initial and final states, read as follows:

$$
e_{ \pm 1}=e_{ \pm 1}^{\prime}=\mp \frac{1}{\sqrt{2}}(0,1, \pm \imath, 0)
$$

while the longitudinal polarization vector in the initial state is

$$
e_{0}=\frac{1}{m_{d}}\left(-K, 0,0, \sqrt{m_{d}^{2}+K^{2}}\right)
$$

and in the final state is

$$
e_{0}^{\prime}=\frac{1}{m_{d}}\left(K, 0,0, \sqrt{m_{d}^{2}+K^{2}}\right)
$$

\section{B Front-form Dirac spinors and matrix elements of $\gamma$ matrices}

Adopting the following representation for the $\gamma$ matrices

$$
\gamma^{0}=\left\|\begin{array}{ll}
0 & 1 \\
1 & 0
\end{array}\right\|, \quad \gamma^{5}=\left\|\begin{array}{cc}
1 & 0 \\
0 & -1
\end{array}\right\|, \quad \gamma^{i}=\left\|\begin{array}{cc}
0 & -\sigma_{i} \\
\sigma_{i} & 0
\end{array}\right\|,
$$

with $i=1,2,3$ and $\sigma_{i}$ the Pauli matrices, the front-form Dirac spinor $w(p, \sigma)$ can be written as

$$
w(p, \sigma)=\sqrt{m}\left\|\begin{array}{c}
\beta(g) \chi(\sigma) \\
{\left[\beta(g)^{-1}\right]^{\dagger} \chi(\sigma)}
\end{array}\right\|,
$$

where $\chi(\sigma)$ is the ordinary spin $1 / 2$ spinor describing the state with spin projection on the $z$ axis equal to $\sigma$ and the matrix $\beta(g)$ has the components

$$
\beta_{11}=\beta_{22}^{-1}=2^{1 / 4}\left(g^{+}\right)^{1 / 2}, \quad \beta_{12}=0, \quad \beta_{21}=\left(g_{x}+g_{y}\right) \beta_{22},
$$


with $g=p / m$.

One can immediately obtain

$$
\bar{w}\left(p^{\prime}, \sigma^{\prime}\right) w(p, \sigma)=\frac{1}{\sqrt{p^{+} p^{\prime+}}}\left\langle\sigma^{\prime}\left|\left[m\left(p^{+}+p^{+}\right)-\imath \hat{\sigma}_{x}\left(p^{+} p_{y}^{\prime}-p^{\prime+} p_{y}\right)+\imath \hat{\sigma}_{y}\left(p^{+} p_{x}^{\prime}-p^{\prime+} p_{x}\right)\right]\right| \sigma\right\rangle
$$

with normalization

$$
\bar{w}\left(p, \sigma^{\prime}\right) w(p, \sigma)=\frac{1}{p^{+}}\left\langle\sigma^{\prime}\left|m 2 p^{+}\right| \sigma\right\rangle=2 m \delta_{\sigma \sigma^{\prime}} .
$$

The matrix elements of the $\gamma$ matrices, needed for the calculation of the deuteron form factors, are

$$
\begin{gathered}
\bar{w}\left(p^{\prime}, \sigma^{\prime}\right) \gamma^{+} w(p, \sigma)=2 \sqrt{p^{+} p^{\prime+}} \delta_{\sigma \sigma^{\prime}} \\
\bar{w}\left(p^{\prime}, \sigma^{\prime}\right) \gamma_{x} w(p, \sigma)=\frac{1}{\sqrt{p^{+} p^{\prime+}}}\left\langle\sigma^{\prime}\left|\left[\imath m q^{+} \hat{\sigma}_{y}+p^{+} p_{x}^{\prime}+p^{\prime+} p_{x}+\imath \hat{\sigma}_{z}\left(p^{\prime+} p_{y}-p^{+} p_{y}^{\prime}\right)\right]\right| \sigma\right\rangle .
\end{gathered}
$$

In our special Breit frame Eqs. (62) and (65) become :

$$
\begin{array}{r}
\bar{w}\left(p^{\prime}, \sigma^{\prime}\right) w(p, \sigma)=\frac{1}{\sqrt{p^{+} p^{\prime+}}}\left\langle\sigma^{\prime}\left|\left[m\left(p^{+}+p^{++}\right)+\imath q^{+}\left(\hat{\sigma}_{x} k_{y}-\hat{\sigma}_{y} k_{x}\right)\right]\right| \sigma\right\rangle \\
\bar{w}\left(p^{\prime}, \sigma^{\prime}\right) \gamma_{x} w(p, \sigma)=\frac{1}{\sqrt{p^{+} p^{\prime+}}}\left\langle\sigma^{\prime}\left|\left[\imath m q^{+} \hat{\sigma}_{y}+\left(p^{+}+p^{\prime+}\right) k_{x}+\imath \hat{\sigma}_{z} k_{y} q^{+}\right]\right| \sigma\right\rangle .
\end{array}
$$

\section{Generalized Melosh matrices for the deuteron wave function}

The generalized Melosh matrices for the deuteron wave function have been defined in Sect. 4 as the matrices

$$
C_{i}(\boldsymbol{k})+\imath \hat{\boldsymbol{\sigma}} \cdot \boldsymbol{D}_{i}(\boldsymbol{k})=v\left(\boldsymbol{k}, \vec{s}_{1}\right)^{-1} \hat{\sigma}_{i} \hat{\sigma}_{y}\left[v\left(-\boldsymbol{k}, \vec{s}_{2}\right)\right]^{*}, \quad i=1,2,3 .
$$

From the expression $(29)$ for the matrix $v(\boldsymbol{k}, \vec{s})$ one obtains

$$
\begin{aligned}
& C_{i}(\boldsymbol{k})=\mathcal{N}\left[\delta_{2 i} m+\frac{k_{y} k_{i}}{m+\omega(k)}\right] \\
& {\left[\boldsymbol{D}_{i}(\boldsymbol{k})\right]_{x}=\mathcal{N}\left[-\delta_{3 i} m+\frac{k_{z} k_{i}-\delta_{3 i} k^{2}}{m+\omega(k)}\right]} \\
& {\left[\boldsymbol{D}_{i}(\boldsymbol{k})\right]_{y}=\mathcal{N}\left(\boldsymbol{e}_{z} \wedge \boldsymbol{k}\right)_{i}} \\
& {\left[\boldsymbol{D}_{i}(\boldsymbol{k})\right]_{z}=\mathcal{N}\left[\delta_{1 i} m+\frac{k_{x} k_{i}}{m+\omega(k)}\right]}
\end{aligned}
$$

where

$$
\mathcal{N}=\frac{1}{M_{0} \sqrt{\xi(1-\xi)}}=\frac{1}{\sqrt{m^{2}+k_{\perp}^{2}}}
$$




\section{Deuteron magnetic and quadrupole moments}

In this Appendix we illustrate the main steps for the calculations of the deuteron magnetic and quadrupole moments from Eqs. (22, 23). To this end, expansions in $\kappa=\sqrt{\tau}=Q /\left(2 m_{d}\right)$ of the quantities $a$ and $b$ (Eq. (44)) up to the first order

$$
a=2, \quad b=2 \frac{\kappa}{\xi}
$$

and of the quantities $\xi$ and $k_{z}$ (Eq. (45)) up to the second order

$$
\xi=\xi^{\prime}-2 \kappa\left(1-\xi^{\prime}\right)-2 \kappa^{2}\left(1-\xi^{\prime}\right), \quad k_{z}=k_{z}^{\prime}-\frac{\kappa \omega\left(k^{\prime}\right)}{\xi^{\prime}}+\omega\left(k^{\prime}\right) \frac{\kappa^{2}}{2 \xi^{\prime 2}}\left(4 \xi^{\prime}-3\right)
$$

will be needed, since the intrinsic moment in the final state, $\vec{k}^{\prime}$, is the integration variable in the integrals for the calculation of the current matrix elements.

\section{D.1 Magnetic moment}

The deuteron magnetic moment is given by Eq. (22)

$$
\mu_{d}=\frac{m_{p}}{\left(\sqrt{2} m_{d}\right)} \lim _{Q \rightarrow 0} \frac{1}{Q}\left[\mathcal{J}_{1,0}^{x}-\mathcal{J}_{0,1}^{x}\right],
$$

where the matrix elements $\mathcal{J}_{1,0}^{x}$ and $\mathcal{J}_{0,1}^{x}$ can be obtained by Eq. (54). Let us preliminarly note that $\mathcal{J}_{1,0}^{x}$ and $\mathcal{J}_{0,1}^{x}$ have the same expression, but for the exchange of the role of initial and final variables in $F_{i, j}(\boldsymbol{k}), F_{i^{\prime}, j^{\prime}}\left(\boldsymbol{k}^{\prime}\right)$, and in the quantity between curly brackets in Eq. (54) (we recall that only the real part of $\left(\vec{e}_{+1}\right)_{j}$ gives a non vanishing contribution to the matrix elements). In order to obtain the magnetic moment, one can expand $\left[\mathcal{J}_{1,0}^{x}-\mathcal{J}_{0,1}^{x}\right]$ as a function of $\kappa$, and consider only the terms which are linear in $\kappa$ (indeed, because of Eqs. (45) and (46), the current matrix elements are functions of $\kappa$ ). As a first step, by using Eq. (75) and Eq. (76), we expand the quantity between curly brackets in Eq. (54) at the first order in $\kappa$. We obtain a term independent of $\kappa$ and a term linear in $\kappa$, which is identical, but with opposite signs, for the two matrix elements $\mathcal{J}_{1,0}^{x}$ and $\mathcal{J}_{0,1}^{x}$. It is clear that in correspondence to the latter term one can evaluate the radial wave functions in Eq. (54) with the same argument $\boldsymbol{k}$. After an integration over the polar angle $\phi[\boldsymbol{k} \equiv(k, \theta, \phi)]$ one has:

$$
\begin{aligned}
& \mu_{d}=-\lim _{Q \rightarrow 0} \frac{m_{p}\left[\mathcal{F}-\mathcal{F}^{\prime}\right]}{2 Q m_{d}}+ \\
& \frac{\pi m m_{p}}{m_{d}^{2}} \int_{0}^{\infty} d\left(k_{\perp}^{2}\right) \int_{-\infty}^{\infty} \frac{d k_{z}}{\xi^{2}} f_{m}^{i s} \chi_{0}(k)\left[\chi_{0}(k)\left(1+\frac{k_{\perp}^{2}}{2 m(\omega(k)+m)}\right)+3 \varphi_{2}(k) \frac{k^{2}+k_{z}^{2}}{2 \sqrt{2} k^{2}}\right]+ \\
& \frac{m_{p} \pi}{2 m m_{d}^{2}} \int_{0}^{\infty} d\left(k_{\perp}^{2}\right) k_{\perp}^{2} \int_{-\infty}^{\infty} \frac{d k_{z}}{\xi^{2}} \chi_{0}(k)\left[\chi_{0}(k)+\frac{3}{\sqrt{2}} \varphi_{2}(k)\right]\left[f_{m}^{i s}-f_{e}^{i s} \frac{\omega(k)}{\omega(k)+m}\right]
\end{aligned}
$$


where the first term and the last two terms correspond to the zero and first order terms in the expansion of the curly bracket of Eq. (54), respectively. In Eq. (78), $\mathcal{F}$ is given by the following expression

$$
\begin{aligned}
& \mathcal{F}=3 \pi \int_{0}^{\infty} d\left(k_{\perp}^{2}\right) k_{\perp}^{2} \int_{-\infty}^{\infty} \frac{d k_{z}^{\prime}}{\xi}\left[\frac{\omega(k)}{\omega\left(k^{\prime}\right)}\right]^{1 / 2} f_{e}^{i s} . \\
& {\left[\chi_{0}(k) \varphi_{2}\left(k^{\prime}\right) \frac{k_{z}^{\prime}}{\sqrt{2} k^{\prime 2}}+\chi_{0}\left(k^{\prime}\right) \varphi_{2}(k) \frac{k_{z}}{\sqrt{2} k^{2}}+\frac{3 k_{z}\left(k_{\perp}^{2}+k_{z} k_{z}^{\prime}\right)}{2 k^{2} k^{\prime 2}} \varphi_{2}(k) \varphi_{2}\left(k^{\prime}\right)\right]}
\end{aligned}
$$

and, according to the observation at the beginning of this subsection, $\mathcal{F}^{\prime}$ has the same expression, but for the exchange of $\boldsymbol{k}$ and $\boldsymbol{k}^{\prime}$ in the quantity between square brackets.

The limit in Eq. (78) can be easily handled and one obtains the final result

$$
\begin{aligned}
& \mu_{d}=8 \pi \frac{m m_{p}}{m_{d}^{2}} \int_{0}^{\infty} k^{2} d k \int_{0}^{1} d(\cos \theta) \frac{\left[(\omega(k))^{2}+k_{z}^{2}\right]}{\left(m^{2}+k_{\perp}^{2}\right)^{2}} . \\
& \left\{\frac{9 \omega(k)}{4 m}\left[\varphi_{2}(k)\right]^{2}\left(1-\cos ^{2} \theta\right)+f_{m}^{i s} \chi_{0}(k)\left[\chi_{0}(k)\left(2+\frac{k_{\perp}^{2}}{m(\omega(k)+m)}\right)+3 \varphi_{2}(k) \frac{\left(1+\cos ^{2} \theta\right)}{\sqrt{2}}\right]+\right. \\
& \left.\chi_{0}(k) \frac{k_{\perp}^{2}}{m^{2}}\left[\chi_{0}(k)+\frac{3}{\sqrt{2}} \varphi_{2}(k)\right]\left[f_{m}^{i s}-\frac{\omega(k)}{\omega(k)+m}\right]\right\} .
\end{aligned}
$$

In Eqs. (78, 80) the nucleon form factors $f_{e}^{i s}$ and $f_{m}^{i s}$ have to be evaluated in the limit $Q \rightarrow 0$, i.e. $f_{e}^{i s}(0)=1, f_{m}^{i s}(0)=0.8797$.

The nonrelativistic result for $\mu_{d}$ can be immediately recovered from Eq. (80) in the limit $m \rightarrow \infty$.

\section{D.2 Quadrupole moment}

The quadrupole form factor (see Eq. (21)) is given by

$$
G_{Q}=\frac{\sqrt{2} m_{d}}{Q^{2}} \frac{\left[\mathcal{J}_{0,0}^{+}-\mathcal{J}_{1,1}^{+}\right]}{\sqrt{1+\tau}}
$$

The proper combination $\left(\mathcal{J}_{0,0}^{+}-\mathcal{J}_{1,1}^{+}\right)$of the matrix elements of $\mathcal{J}^{+}$can be directly calculated from Eq. (53) by using Eqs. (38), (51) and the explicit expressions for the the quantities $C_{i}(\boldsymbol{k}), \boldsymbol{D}_{i}(\boldsymbol{k})$ given in Appendix C. One obtains

$$
\mathcal{J}_{0,0}^{+}-\mathcal{J}_{1,1}^{+}=m_{d} \sqrt{2} \int\left[\frac{\omega(k) \xi^{\prime}}{\omega\left(k^{\prime}\right) \xi}\right]^{1 / 2}\left[\left(f_{e}^{i s}-f_{m}^{i s}\right) b k_{\perp} \frac{\left(b k_{\perp} E-a m H\right)}{a^{2} m^{2}+b^{2} k_{\perp}^{2}}-f_{e}^{i s} E\right] d \vec{k}^{\prime}
$$

where

$$
E=\frac{1}{2} \chi_{0}(k) \chi_{0}\left(k^{\prime}\right)\left[1-\cos \left(\varphi-\varphi^{\prime}\right)\right]+
$$




$$
\begin{aligned}
& \frac{3 \varphi_{2}(k) \chi_{0}\left(k^{\prime}\right)}{\sqrt{2} k^{2}}\left[\left(\frac{1}{2} k_{\perp}^{2}-k_{z}^{2}\right) \cos \left(\varphi-\varphi^{\prime}\right)+\frac{3}{2} k_{z} k_{\perp} \sin \left(\varphi-\varphi^{\prime}\right)\right]+ \\
& \frac{3 \varphi_{2}\left(k^{\prime}\right) \chi_{0}(k)}{\sqrt{2} k^{\prime 2}}\left[\left(\frac{1}{2} k_{\perp}^{2}-k_{z}^{\prime 2}\right) \cos \left(\varphi-\varphi^{\prime}\right)-\frac{3}{2} k_{z}^{\prime} k_{\perp} \sin \left(\varphi-\varphi^{\prime}\right)\right]+ \\
& \frac{9 \varphi_{2}(k) \varphi_{2}\left(k^{\prime}\right)}{2 k^{2} k^{\prime 2}}\left(\frac{1}{2} k_{\perp}^{2}-k_{z} k_{z}^{\prime}\right)\left[\left(k_{\perp}^{2}+k_{z} k_{z}^{\prime}\right) \cos \left(\varphi-\varphi^{\prime}\right)+\left(k_{z}-k_{z}^{\prime}\right) k_{\perp} \sin \left(\varphi-\varphi^{\prime}\right)\right]
\end{aligned}
$$

and

$$
\begin{aligned}
& H=\frac{1}{2} \chi_{0}(k) \chi_{0}\left(k^{\prime}\right) \sin \left(\varphi-\varphi^{\prime}\right)+ \\
& \frac{3 \varphi_{2}(k) \chi_{0}\left(k^{\prime}\right)}{\sqrt{2} k^{2}}\left[\left(k_{z}^{2}-\frac{1}{2} k_{\perp}^{2}\right) \sin \left(\varphi-\varphi^{\prime}\right)+\frac{3}{2} k_{z} k_{\perp} \cos \left(\varphi-\varphi^{\prime}\right)\right]+ \\
& \left.\frac{3 \varphi_{2}\left(k^{\prime}\right) \chi_{0}(k)}{\sqrt{2} k^{\prime 2}}\right)\left[\left(k_{z}^{\prime 2}-\frac{1}{2} k_{\perp}^{2}\right) \sin \left(\varphi-\varphi^{\prime}\right)-\frac{3}{2} k_{z}^{\prime} k_{\perp} \cos \left(\varphi-\varphi^{\prime}\right)\right]+ \\
& \frac{9 \varphi_{2}(k) \varphi_{2}\left(k^{\prime}\right)}{2 k^{2} k^{\prime 2}}\left(k_{z} k_{z}^{\prime}-\frac{1}{2} k_{\perp}^{2}\right)\left[\left(k_{\perp}^{2}+k_{z} k_{z}^{\prime}\right) \sin \left(\varphi-\varphi^{\prime}\right)+\left(k_{z}-k_{z}^{\prime}\right) k_{\perp} \cos \left(\varphi-\varphi^{\prime}\right)\right]
\end{aligned}
$$

The angle $\varphi^{\prime}$ is defined by Eq. (31) with $\boldsymbol{k}$ replaced by $\boldsymbol{k}^{\prime}$.

The expression for $G_{Q}$ given by Eqs. (81,82, 83, 84) holds at any value of $Q^{2}$. For the evaluation of the quadrupole moment

$$
\begin{aligned}
& Q_{d}=\frac{\sqrt{2}}{m_{d}} \lim _{Q \rightarrow 0} \frac{1}{Q^{2}}\left[\mathcal{J}_{0,0}^{+}-\mathcal{J}_{1,1}^{+}\right]= \\
& \lim _{Q \rightarrow 0} \frac{2}{Q^{2}} \int\left[\frac{\omega(k) \xi^{\prime}}{\omega\left(k^{\prime}\right) \xi}\right]^{1 / 2}\left[\left(f_{e}^{i s}-f_{m}^{i s}\right) b k_{\perp} \frac{\left(b k_{\perp} E-a m H\right)}{a^{2} m^{2}+b^{2} k_{\perp}^{2}}-f_{e}^{i s} E\right] d \vec{k}^{\prime}
\end{aligned}
$$

an expansion of $\left[\mathcal{J}_{0,0}^{+}-\mathcal{J}_{1,1}^{+}\right]$at the second order in $\kappa$ is needed.

Let us note that at the first order in $\kappa$ one has

$$
\varphi^{\prime}-\varphi=\frac{k_{\perp} \kappa}{\xi(\omega(k)+m)}
$$

and, as a consequence, the quantity $H$ is of the first order in $\kappa$

$$
H=\kappa H_{1}+\mathcal{O}\left(\kappa^{2}\right)
$$

with

$$
\begin{aligned}
& H_{1}=\frac{k_{\perp}}{2 \xi}\left\{\frac{1}{\omega(k)+m} \cdot\right. \\
& {\left[-\left[\chi_{0}(k)\right]^{2}+\frac{\left(k_{\perp}^{2}-2 k_{z}^{2}\right)}{2 k^{2}} 3 \varphi_{2}(k)\left(2 \sqrt{2} \varphi_{0}(k)+\varphi_{2}(k)+3 \varphi_{2}(k) \frac{\omega(k)(\omega(k)+m)}{k^{2}}\right)\right]-} \\
& \left.\frac{9 \omega(k)}{\sqrt{2} k^{2}}\left[\varphi_{2}(k) \chi_{0}(k) \frac{\left(k_{\perp}^{2}-k_{z}^{2}\right)}{k^{2}}+\frac{k_{z}^{2}}{k}\left(\varphi_{0}(k) \frac{\partial \varphi_{2}(k)}{\partial k}-\varphi_{2}(k) \frac{\partial \varphi_{0}(k)}{\partial k}\right)\right]\right\}
\end{aligned}
$$


Since $b$ is also of the first order in $\kappa$ (see Eq. (75)), in Eq. (85) one can take $a=2$ and disregard $b^{2} k_{\perp}^{2}$ with respect to $a^{2} m^{2}$ in the limit $Q^{2} \rightarrow 0$. As a result one has

$$
\begin{aligned}
& Q_{d}=\lim _{Q \rightarrow 0} \frac{2}{Q^{2}} \int\left[\frac{\omega(k) \xi^{\prime}}{\omega\left(k^{\prime}\right) \xi}\right]^{1 / 2}\left[\frac{Q^{2}}{m_{d}^{2}}\left(f_{e}^{i s}-f_{m}^{i s}\right) k_{\perp} \frac{\left(k_{\perp} E-m \xi H_{1}\right)}{4 m^{2} \xi^{2}}-f_{e}^{i s} E\right] d \vec{k}^{\prime}= \\
& =Q_{d 1}+Q_{d 2}
\end{aligned}
$$

where

$$
\begin{aligned}
Q_{d 1} & =\frac{2}{m_{d}^{2}} \int\left[f_{e}^{i s}(0)-f_{m}^{i s}(0)\right] k_{\perp} \frac{\left(k_{\perp} E_{0}-m \xi H_{1}\right)}{4 m^{2} \xi^{2}} d \vec{k} \\
Q_{d 2} & =-\lim _{Q \rightarrow 0} \frac{2}{Q^{2}} \int\left[\frac{\omega(k) \xi^{\prime}}{\omega\left(k^{\prime}\right) \xi}\right]^{1 / 2} f_{e}^{i s} E d \vec{k}^{\prime}
\end{aligned}
$$

In the integral of Eq. (90) each quantity has been evaluated at $Q^{2}=0$ (i.e., $\xi=\xi^{\prime}, k_{z}=k_{z}^{\prime}$ ) and

$$
E_{0}=E\left(Q^{2}=0\right)=\left(\frac{1}{2} k_{\perp}^{2}-k_{z}^{2}\right) \frac{3 \varphi_{2}(k)\left(2 \sqrt{2} \varphi_{0}(k)+\varphi_{2}(k)\right)}{2 k^{2}} .
$$

To evaluate $Q_{d}$ we need an expansion of the integral in Eq. (91) up to the second order in $Q$. By using the expansions of $\xi$ and $k_{z}$ up to the second order in $\kappa$ given in Eq. (76), one obtains

$$
\begin{aligned}
& {\left[\frac{\omega(k) \xi^{\prime}}{\omega\left(k^{\prime}\right) \xi}\right]^{1 / 2}=1+\kappa \Omega_{1}+\frac{\kappa^{2}}{2} \Omega_{2}} \\
& E=E_{0}+\kappa E_{1}+\frac{\kappa^{2}}{2} E_{2}
\end{aligned}
$$

where

$$
\begin{aligned}
& \Omega_{1}=-\frac{4 \xi^{\prime}-3}{2 \xi^{\prime}}, \quad \Omega_{2}=\frac{16 \xi^{\prime 2}-36 \xi^{\prime}+21}{4 \xi^{\prime 2}} \\
& E_{1}=\frac{3 \omega\left(k^{\prime}\right) k_{z}^{\prime}}{\sqrt{2} \xi^{\prime} k^{\prime 3}}\left[\frac{3 k_{\perp}^{2}}{k^{\prime}} \varphi_{2}\left(k^{\prime}\right)\left(\varphi_{0}\left(k^{\prime}\right)+\frac{\varphi_{2}\left(k^{\prime}\right)}{2 \sqrt{2}}\right)-\right. \\
& \left.\left(\frac{k_{\perp}^{2}}{2}-k_{z}^{\prime 2}\right)\left(\varphi_{0}\left(k^{\prime}\right) \frac{\partial \varphi_{2}\left(k^{\prime}\right)}{\partial k^{\prime}}+\varphi_{2}\left(k^{\prime}\right) \frac{\partial \varphi_{0}\left(k^{\prime}\right)}{\partial k^{\prime}}+\frac{\varphi_{2}\left(k^{\prime}\right)}{\sqrt{2}} \frac{\partial \varphi_{2}\left(k^{\prime}\right)}{\partial k^{\prime}}\right)\right] \\
& E_{2}=\frac{k_{\perp}^{2}}{k^{\prime 2} \xi^{\prime 2}\left(\omega\left(k^{\prime}\right)+m\right)^{2}}\left\{\frac{k^{\prime 2}\left[\chi_{0}\left(k^{\prime}\right)\right]^{2}}{2}-3 \varphi_{2}\left(k^{\prime}\right)\left(\frac{k_{\perp}^{2}}{2}-k_{z}^{\prime 2}\right)\left(\sqrt{2} \chi_{0}\left(k^{\prime}\right)+\frac{3}{2} \varphi_{2}\left(k^{\prime}\right)\right)+\right. \\
& \frac{9 \omega\left(k^{\prime}\right)}{\sqrt{2} k^{\prime}}\left(\omega\left(k^{\prime}\right)+m\right)\left[\frac{\varphi_{2}\left(k^{\prime}\right)}{k^{\prime}} \chi_{0}\left(k^{\prime}\right)\left(k_{\perp}^{2}-k_{z}^{\prime 2}\right)+\right.
\end{aligned}
$$




$$
\begin{aligned}
& \left.\left.k_{z}^{\prime 2}\left(\varphi_{0}\left(k^{\prime}\right) \frac{\partial \varphi_{2}\left(k^{\prime}\right)}{\partial k^{\prime}}-\varphi_{2}\left(k^{\prime}\right) \frac{\partial \varphi_{0}\left(k^{\prime}\right)}{\partial k^{\prime}}\right)+\sqrt{2} \frac{\left[\varphi_{2}\left(k^{\prime}\right)\right]^{2}}{k^{\prime}}\left(\frac{k_{\perp}^{2}}{2}-k_{z}^{\prime 2}\right)\right]\right\}+ \\
& \frac{3 \omega\left(k^{\prime}\right) k_{z}^{\prime}}{\sqrt{2} \xi^{\prime 2} k^{\prime 3}}\left(4 \xi^{\prime}-3\right)\left\{\left(\frac{k_{\perp}^{2}}{2}-k_{z}^{\prime 2}\right)\left[\varphi_{2}\left(k^{\prime}\right) \frac{\partial \varphi_{0}\left(k^{\prime}\right)}{\partial k^{\prime}}+\varphi_{0}\left(k^{\prime}\right) \frac{\partial \varphi_{2}\left(k^{\prime}\right)}{\partial k^{\prime}}+\frac{\varphi_{2}\left(k^{\prime}\right)}{\sqrt{2}} \frac{\partial \varphi_{2}\left(k^{\prime}\right)}{\partial k^{\prime}}\right]-\right. \\
& \left.\frac{3 k_{\perp}^{2}}{k^{\prime}} \varphi_{2}\left(k^{\prime}\right)\left(\varphi_{0}\left(k^{\prime}\right)+\frac{\varphi_{2}\left(k^{\prime}\right)}{2 \sqrt{2}}\right)\right\}+ \\
& \frac{3\left[\omega\left(k^{\prime}\right)\right]^{2}}{\sqrt{2} \xi^{\prime 2} k^{\prime 4}}\left\{( \frac { k _ { \perp } ^ { 2 } } { 2 } - k _ { z } ^ { \prime 2 } ) \left[\varphi_{2}\left(k^{\prime}\right)\left(\frac{k_{\perp}^{2}}{k^{\prime}} \frac{\partial \varphi_{0}\left(k^{\prime}\right)}{\partial k^{\prime}}+k_{z}^{\prime 2} \frac{\partial^{2} \varphi_{0}\left(k^{\prime}\right)}{\partial k^{\prime 2}}\right)+\right.\right. \\
& \left.\varphi_{0}\left(k^{\prime}\right)\left(\frac{k_{\perp}^{2}}{k^{\prime}} \frac{\partial \varphi_{2}\left(k^{\prime}\right)}{\partial k^{\prime}}+k_{z}^{\prime 2} \frac{\partial^{2} \varphi_{2}\left(k^{\prime}\right)}{\partial k^{\prime 2}}\right)+\frac{k_{z}^{\prime 2}}{\sqrt{2}} \varphi_{2}\left(k^{\prime}\right) \frac{\partial^{2} \varphi_{2}\left(k^{\prime}\right)}{\partial k^{\prime 2}}\right]+ \\
& \frac{k_{\perp}^{2}}{k^{\prime}}\left[\frac{3}{k^{\prime}} \varphi_{0}\left(k^{\prime}\right) \varphi_{2}\left(k^{\prime}\right)\left(3 k_{z}^{\prime 2}-k_{\perp}^{2}\right)-6 k_{z}^{\prime 2} \varphi_{0}\left(k^{\prime}\right) \frac{\partial \varphi_{2}\left(k^{\prime}\right)}{\partial k^{\prime}}+\frac{3 \sqrt{2} k_{z}^{\prime 2}}{k^{\prime}}\left[\varphi_{2}\left(k^{\prime}\right)\right]^{2}+\right. \\
& \left.\left.\sqrt{2}\left(\frac{k_{\perp}^{2}}{4}-2 k_{z}^{\prime 2}\right) \varphi_{2}\left(k^{\prime}\right) \frac{\partial \varphi_{2}\left(k^{\prime}\right)}{\partial k^{\prime}}\right]\right\}
\end{aligned}
$$

Furthermore, because of Eq. (46), one has

$$
f_{e}^{i s}\left(\left(p_{1}^{\prime}-p_{1}\right)^{2}\right)=1+Q^{2}\left[\frac{d f_{e}^{i s}\left(\left(p_{1}^{\prime}-p_{1}\right)^{2}\right)}{d\left(Q^{2}\right)}\right]_{Q^{2}=0}=1-\frac{\left(r_{e}^{i s}\right)^{2}}{3} \frac{2 \kappa^{2}\left(m^{2}+k_{\perp}^{2}\right)}{\xi \xi^{\prime}}
$$

where

$$
\left(r_{e}^{i s}\right)^{2}=6\left[\frac{d f_{e}^{i s}\left(\left(p_{1}^{\prime}-p_{1}\right)^{2}\right)}{d\left(\left(p_{1}^{\prime}-p_{1}\right)^{2}\right)}\right]_{Q^{2}=0}=r_{e p}^{2}+r_{e n}^{2}
$$

is the sum of the squares of the proton and neutron charge mean square radii (let us recall that $\left.\left(p_{1}^{\prime}-p_{1}\right)^{2} \leq 0\right)$. Then, since only the second order terms in the expansion of the integral in Eq. (91) can give a contribution to $Q_{d}$, one obtains

$$
Q_{d 2}=-\frac{1}{4 m_{d}^{2}} \int\left[\Omega_{2} E_{0}+E_{2}+2 \Omega_{1} E_{1}-4 E_{0} \frac{\left(r_{e}^{i s}\right)^{2}}{3} \frac{\left(m^{2}+k_{\perp}^{2}\right)}{\xi^{2}}\right] d \vec{k}
$$

where each quantity has to be evaluated at $Q^{2}=0$.

\section{References}

[1] L.A. Kondratyuk and M.I. Strikman, Nucl. Phys. A 426, 575 (1984).

[2] E. Hummel and J.A. Tjon, Phys. Rev. C 49, 21 (1994).

[3] J.W. Van Orden, N. Devine and F. Gross, Phys. Rev. Lett. 75, 4369 (1995). 
[4] J. Carbonell, B. Desplanques, V.A. Karmanov and J.-F. Mathiot, Phys. Rep. 300, 215 (1998); J. Carbonell, V.A. Karmanov, Eur. Phys. J. A 6, 9 (1999).

[5] L.P. Kaptari, A.Yu. Umnikov, S.G. Bondarenko, K.Yu. Kazakov, F.C. Khanna and B. Kämpfer, Phys. Rev. C 54, 986 (1996).

[6] D. R. Phillips, S. J. Wallace and N. K. Devine, Phys. Rev. C58, 2261 (1998); nuclth/9906086.

[7] A. Amghar, N. Aissat, B. Desplanques, Eur. Phys. J. A 1, 85 (1998).

[8] J. Carlson and R. Schiavilla, Rev. Mod. Phys. 70, 743 (1998).

[9] P.L. Chung, F. Coester, B.D. Keister and W.N. Polyzou, Phys. Rev. C 37, 2000 (1988);

P.L. Chung, B.D. Keister, F. Coester, Phys. Rev. C 39, 1544 (1989).

[10] F.M. Lev, E. Pace, G. Salmè, Nucl. Phys. A 641, 229 (1998).

[11] L.C. Alexa et al., Phys. Rev. Lett. 82, 1374 (1999).

[12] D. Abbott et al., Phys. Rev. Lett. 82, 1379 (1999).

[13] F.M. Lev, E. Pace, G. Salmè, Phys. Rev. Lett. 83, 5250 (1999).

[14] P.A.M. Dirac, Rev. Mod. Phys. 21, 392 (1949).

[15] M.V. Terent'ev, Sov. J. Nucl. Phys. 24, 106 (1976).

[16] F.M. Lev, Rivista Nuovo Cimento 16, 1 (1993).

[17] B.D. Keister and W.N. Polyzou, Adv. Nucl. Phys. 21, 225 (1991).

[18] I.L. Grach and L.A. Kondratyuk, Yad. Fiz. 39, 316 (1984).

[19] L.L. Frankfurt, I.L. Grach, L.A. Kondratyuk and M.I. Strikman, Phys. Rev. Lett. 62, 387 (1989).

[20] S.J. Brodsky and J.R. Hiller, Phys. Rev. D 46, 2141 (1992).

[21] L.L. Frankfurt, T. Frederico and M.I. Strikman, Phys. Rev. C 48, 2182 (1993).

[22] T. Frederico and R.-W. Schulze, Phys. Rev. C 54, 2201 (1996).

[23] V.A. Karmanov and A.V. Smirnov, Nucl. Phys. A 546, 691 (1992); Nucl. Phys. A575, 520 (1994).

[24] E.L. Lomon, Ann. Phys. (N.Y.) 125, 309 (1980).

[25] R.B. Wiringa, V.G.J. Stoks and R. Schiavilla, Phys. Rev. C 51, 38 (1995). 
[26] E. Hummel and J. A. Tjon, Phys. Rev. C 42, 423 (1990).

[27] E. Pace, G. Salmè, F. Lev, Few-Body Syst. Suppl. 10, 135 (1999); Proceedings of the Workshop on "Electron Nucleus Scattering", ed. O. Benhar, A. Fabrocini and R. Schiavilla (Edizioni ETS, Pisa, 1999) p. 401; in "Perspectives on Theoretical Nuclear Physics", ed. A. Fabrocini, G. Pisent and S. Rosati (Edizioni ETS, Pisa, 1999) p. 309; Nucl. Phys. A 663-664, 365c (2000); Proceedings of the Second International Conference on "Perspectives in Hadronic Physics", eds. S. Boffi, C. Ciofi degli Atti, M. Giannini (World Scientific Publishing Co., 1999, Singapore) p. 154.

[28] S. Weinberg, "The Quantum Theory of Fields", Cambridge University Press, Cambridge (1995).

[29] V. Glaser and B. Jaksic, Nuovo Cimento 5, 1197 (1957).

[30] E. Pace, G. Salmè, F. Lev, Phys. Rev. C 58, 2655 (1998).

[31] H. Melosh, Phys. Rev. D9, 1095 (1974).

[32] F. Coester, S.C. Pieper and F.J.D. Serduke, Phys. Rev. C 11, 1 (1975).

[33] R.V. Reid, Ann. of Phys. 50, 411 (1968).

[34] R.B. Wiringa, R.A. Smith and T.A. Ainsworth, Phys. Rev. C 29, 1207 (1984).

[35] M. Lacombe et al., Phys. Rev. C 21, 861 (1980).

[36] V.G.J. Stoks, R.A.M. Klomp, C.P.F. Terheggen, J.J. de Swart, Phys. Rev. C 49, 2950 (1994).

[37] R. Machleidt, F. Sammarruca, Y. Song, Phys. Rev. C 53, R1483 (1996).

[38] T.E.O. Ericson and M. Rosa-Clot, Nucl. Phys. A 405, 497 (1983).

[39] S. Klarsfeld, J. Martorell and D.W.L. Sprung, J. Phys. G: Nucl. Phys. 10, L205 (1984).

[40] N.L. Rodning, L.D. Knutson, Phys. Rev. C 41, 898 (1990).

[41] I. Lindgren, in "Alpha-, Beta-, and Gamma-Ray Spectroscopy", ed. K. Siegbahn (NorthHolland, Amsterdam, 1965) Vol. 2, p. 1620.

[42] M. Gari and W. Krümpelmann, Z. Phys. 322, 689 (1985).

[43] M. Gourdin, Il Nuovo Cim. 28, 533 (1963).

[44] S. Galster et al., Nucl. Phys. B 32, 221 (1971).

[45] S. Platchkov et al., Nucl. Phys. A 510, 740 (1990). 
[46] R.G. Arnold et al., Phys. Rev. Lett. 35, 776 (1975).

[47] C.D. Buchanan, M.R. Yearian, Phys. Rev. Lett. 15, 303 (1965).

[48] D. Ganichot, B. Grossetete, Nucl. Phys. A 178, 545 (1972).

[49] S. Auffret et al., Phys. Rev. Lett. 54, 649 (1985).

[50] R. Cramer et al., Z. Phys. C 29, 513 (1985).

[51] P.E. Bosted et al., Phys. Rev. C 42, 38 (1990).

[52] K. McCormick (Jefferson Lab Hall A Collaboration), Fizika B (Zagreb) 8, 55 (1999).

[53] M.E. Schulze et al., Phys. Rev. Lett. 52, 597 (1984).

[54] V.F. Dmitriev, Phys. Lett. B 157, 143 (1985).

[55] R. Gilman et al., Phys. Rev. Lett. 65, 1733 (1990).

[56] I. The et al., Phys. Rev. Lett. 67, 173 (1991); M. Garcon et al., Phys. Rev. C 49, 2516 (1994).

[57] M. Ferro-Luzzi et al., Phys. Rev. Lett. 77, 2630 (1996).

[58] M. Bouwhuis et al., Phys. Rev. Lett. 82, 3755 (1999).

[59] D. Abbott et al., Phys. Rev. Lett. 84, 5053 (2000).

[60] G. Höhler et al., Nucl. Phys. B114, 505 (1975).

[61] B.W. Raichle, C.R. Gould, D.G. Haase, M.L. Seely, J.R. Watson, W. Tornow, W.S. Wilburn, S.I. Penttilä, and G.W. Hoffmann, Phys. Rev. Lett. 83, 2711 (1999).

[62] A. Polls, H. Müther, R. Machleidt and M. Hjorth-Jensen, Phys. Lett. B 432, 1 (1998).

[63] P. Mergell, U-G. Meissner, D. Drechsel, Nucl. Phys. A 596, 367 (1996). 


\begin{tabular}{|c|c|c|c|c|c|c|}
\hline Interaction & $P_{D}$ & $\eta$ & $\mu_{d}^{N R}$ & $\mu_{d}(\mathrm{LPS})$ & $Q_{d}^{N R} \mathrm{fm}^{2}$ & $Q_{d}(\mathrm{LPS}) \mathrm{fm}^{2}$ \\
\hline & & & & & & \\
Exp & & $0.0256(4)$ [40] & & $0.857406(1)$ 41] & & $0.2859(3)$ 38] \\
RSC [33 & 6.47 & 0.0262 & 0.8429 & 0.8611 & 0.2796 & 0.2852 \\
Av14 34] & 6.08 & 0.0265 & 0.8451 & 0.8608 & 0.2860 & 0.2907 \\
Paris [35] & 5.77 & 0.0261 & 0.8469 & 0.8632 & 0.2793 & 0.2841 \\
Av18 250 & 5.76 & 0.0250 & 0.8470 & 0.8635 & 0.2696 & 0.2744 \\
Nijm93 [36 & 5.75 & 0.0252 & 0.8470 & 0.8629 & 0.2706 & 0.2750 \\
RSC93 [36] & 5.70 & 0.0251 & 0.8473 & 0.8637 & 0.2703 & 0.2750 \\
Nijm1 36 & 5.66 & 0.0253 & 0.8475 & 0.8622 & 0.2719 & 0.2758 \\
Nijm2 [36] & 5.64 & 0.0252 & 0.8477 & 0.8652 & 0.2707 & 0.2756 \\
CD-Bonn [37 & 4.83 & 0.0255 & 0.8523 & 0.8670 & 0.2696 & 0.2729 \\
\hline
\end{tabular}

\section{TABLE CAPTION}

Table I. Magnetic moment (in nuclear magnetons) and quadrupole moment for the deuteron, corresponding to different $N-N$ interactions; $\mu_{d}^{N R}$ and $Q_{d}^{N R}$ are the nonrelativistic results, $\mu_{d}$ (LPS) and $Q_{d}$ (LPS) our results; $P_{D}$ is the $D$-state percentage, and $\eta=A_{D} / A_{S}$ the symptotic normalization ratio (this table is taken from Ref. [13], a part from the results for the Nijmegen2 interaction).

Table I F.M. LEV, E. PACE, G. SALMÈ 


\section{FIGURE CAPTIONS}

FIG. 1. (a) Deuteron form factor $A\left(Q^{2}\right)$ obtained using the $R S C N-N$ interaction [33] and the Gari-Krümpelmann nucleon form factors 42]. Solid line: full result of our approach with the Poincaré covariant current operator. Dashed line: the argument of the nucleon form factors, $\left.\left(p_{1}^{\prime}-p_{1}\right)^{2}\right)$, is replaced by $-Q^{2}$. Long-dashed line: nonrelativistic result obtained with exact relativistic relations between deuteron form factors and current matrix elements, within the Breit reference frame where $\hat{q}=\boldsymbol{e}_{z}$ [43], but with nonrelativistic expressions for the matrix elements evaluated in impulse approximation [24]. Experimental data are from Ref. 44] (open squares), Ref. [45] (triangles), Ref. [46] (diamonds), Ref. [11] (full dots) and 112 (open dots). (b) The same as in (a), but for $B\left(Q^{2}\right)$. Experimental data are from Ref. [47] (open dots), Ref. [48] (open squares), Ref. [49] (full diamonds), Ref. [50] (triangles), Ref. [51] (full squares), and [52 (open diamonds). (c) The same as in (a), but for $T_{20}\left(Q^{2}\right)$. Experimental data are from Ref. [53] (open dots), Ref. [54] (full triangles), Ref. [55] (open triangles), Ref. [56] (full dots), Ref. [57 (open squares), Ref. [58 (full squares), and Ref. [59] (diamonds).

FIG. 2. (a) As in Fig. 1 (a), but for the reduced form factor $A\left(Q^{2}\right) /\left(G_{D}^{2} \cdot F\right)$ with $G_{D}=\left(1+Q^{2} / 0.71\right)^{-2}$ and $F=\left(1+Q^{2} / 0.1\right)^{-2.5}$. (b) As in Fig. 1 (b), but for the reduced form factor $\Gamma_{M}\left(Q^{2}\right) /\left(G_{D}^{2} \cdot F_{1}\right)$ with $\Gamma_{M}\left(Q^{2}\right)=\left[G_{M}\left(Q^{2}\right) m_{p} /\left(\mu_{d} m_{d}\right)\right]^{2}$ and $F_{1}=\left(1+Q^{2} / 0.1\right)^{-3}$. Experimental data are as in Fig. 1.

FIG. 3. (a) The deuteron form factor $A\left(Q^{2}\right)$ obtained using our Poincaré covariant current operator, different $N-N$ interactions and the nucleon form factors by Höhler et al. [60]. Solid line: RSC interaction [33]; dashed line: $A V 18$ interaction [25]; dotdashed line: Nijmegen1 interaction; long-dashed line: Nijmegen2 interaction; short-dashed line: Nijmegen93 interaction [36]; dotted line: $C D-$ Bonn interaction [37]. Actually the Nijmegen93 result is very similar to the $A V 18$ one and is not reported in this figure. (b) The same as in (a), but for $B\left(Q^{2}\right)$. (c) The same as in (a), but for $T_{20}\left(Q^{2}\right)$. Experimental data are as in Fig. 1.

FIG. 4. (a) As in Fig. 3 (a), but for the reduced form factor $A\left(Q^{2}\right) /\left(G_{D}^{2} \cdot F\right)$. (b) As in (a), but at low $Q^{2}$. (c) As in Fig. $3(\mathrm{~b})$, but for the reduced form factor $\Gamma_{M}\left(Q^{2}\right) /\left(G_{D}^{2} \cdot F_{1}\right)$. The Nijmegen 1 result is very similar to the $C D-B o n n$ one and is not reported in this figure. Experimental data are as in Fig. 1

FIG. 5. (a) The position of the minimum of $B\left(Q^{2}\right)$, and (b) the position of the second zero of $T_{20}\left(Q^{2}\right)$, corresponding to the Gari-Krümpelmann nucleon form factors 42, vs the nonrelativistic S-state kinetic energy for the deuteron for different realistic interactions.

FIG. 6. (a) The reduced deuteron form factor $A\left(Q^{2}\right) /\left(G_{D}^{2} \cdot F\right)$ obtained with the Nijmegen 2 interaction for different nucleon form factor models. Solid line: nucleon f.f. of Ref. [63]; dashed line: nucleon f.f. of Ref. [60]; dotted line: nucleon f.f. of Ref. [42]. (b) 
As in (a), but for the reduced form factor $\Gamma_{M}\left(Q^{2}\right) /\left(G_{D}^{2} \cdot F_{1}\right)$. (c) As in (a), but for $T_{20}\left(Q^{2}\right)$. Experimental data are as in Fig. 1. 


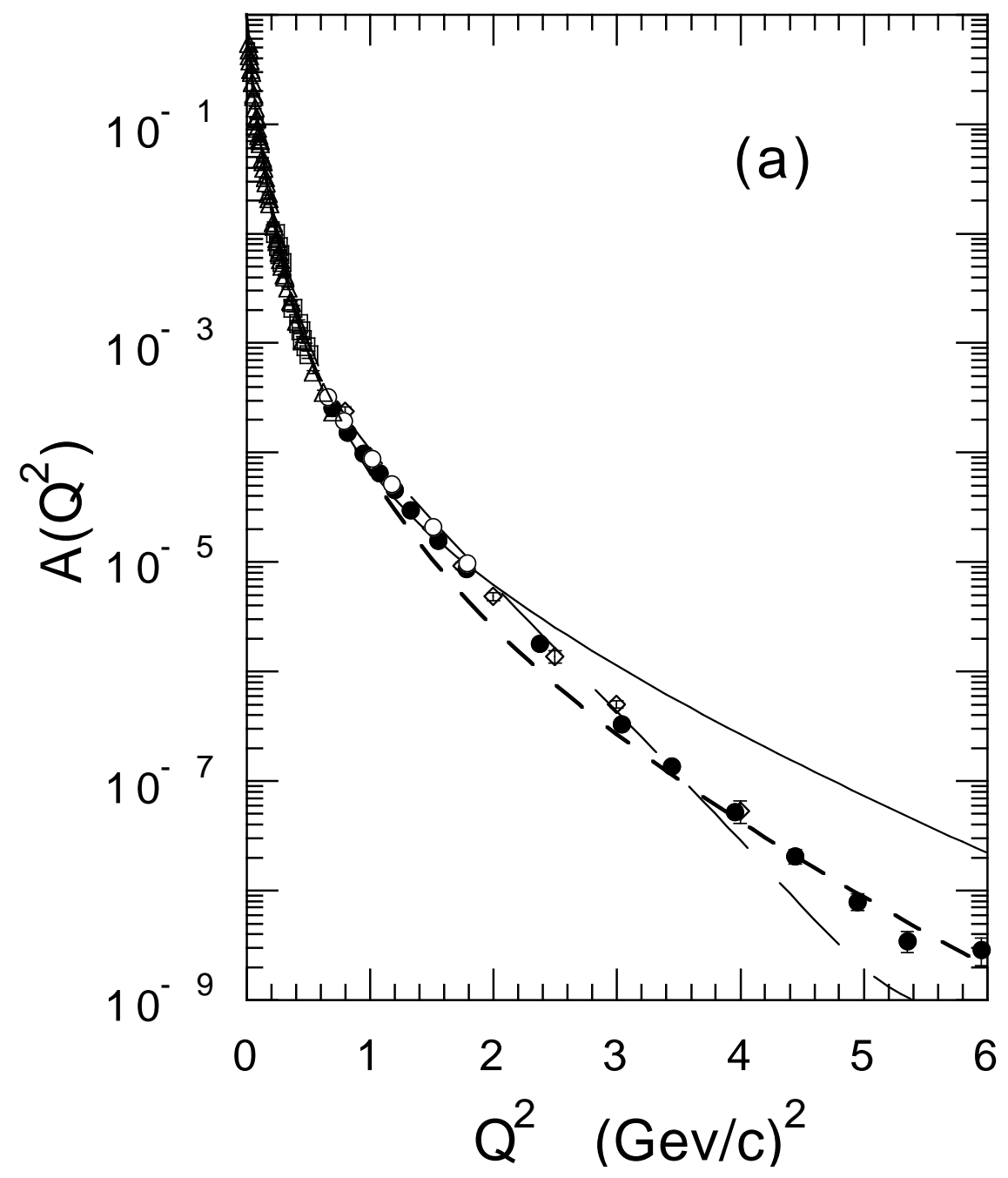

Fig. 1a F.M. LEV, E. PACE, G. SALMÈ 


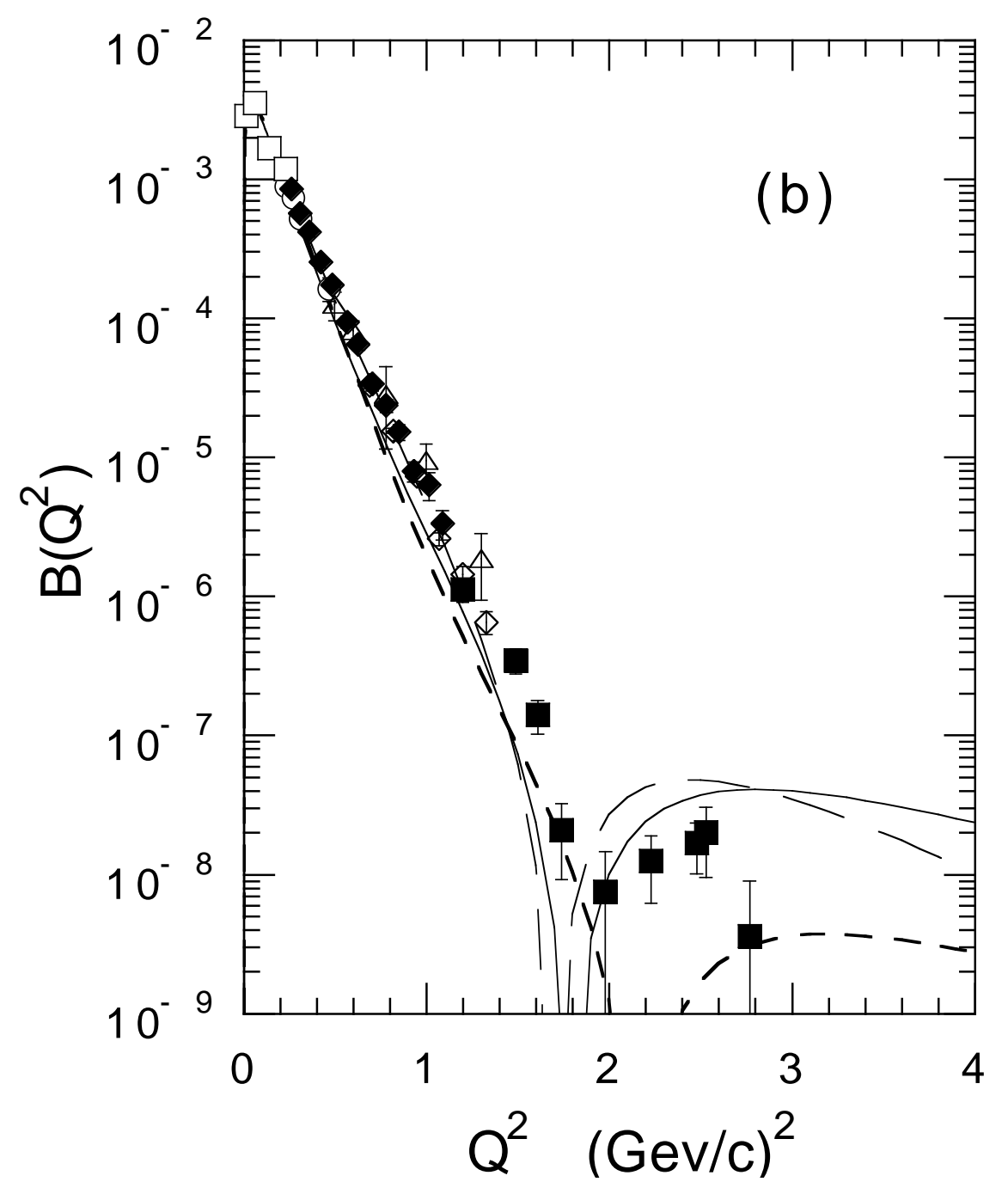

Fig. 1b F.M. LEV, E. PACE, G. SALME 


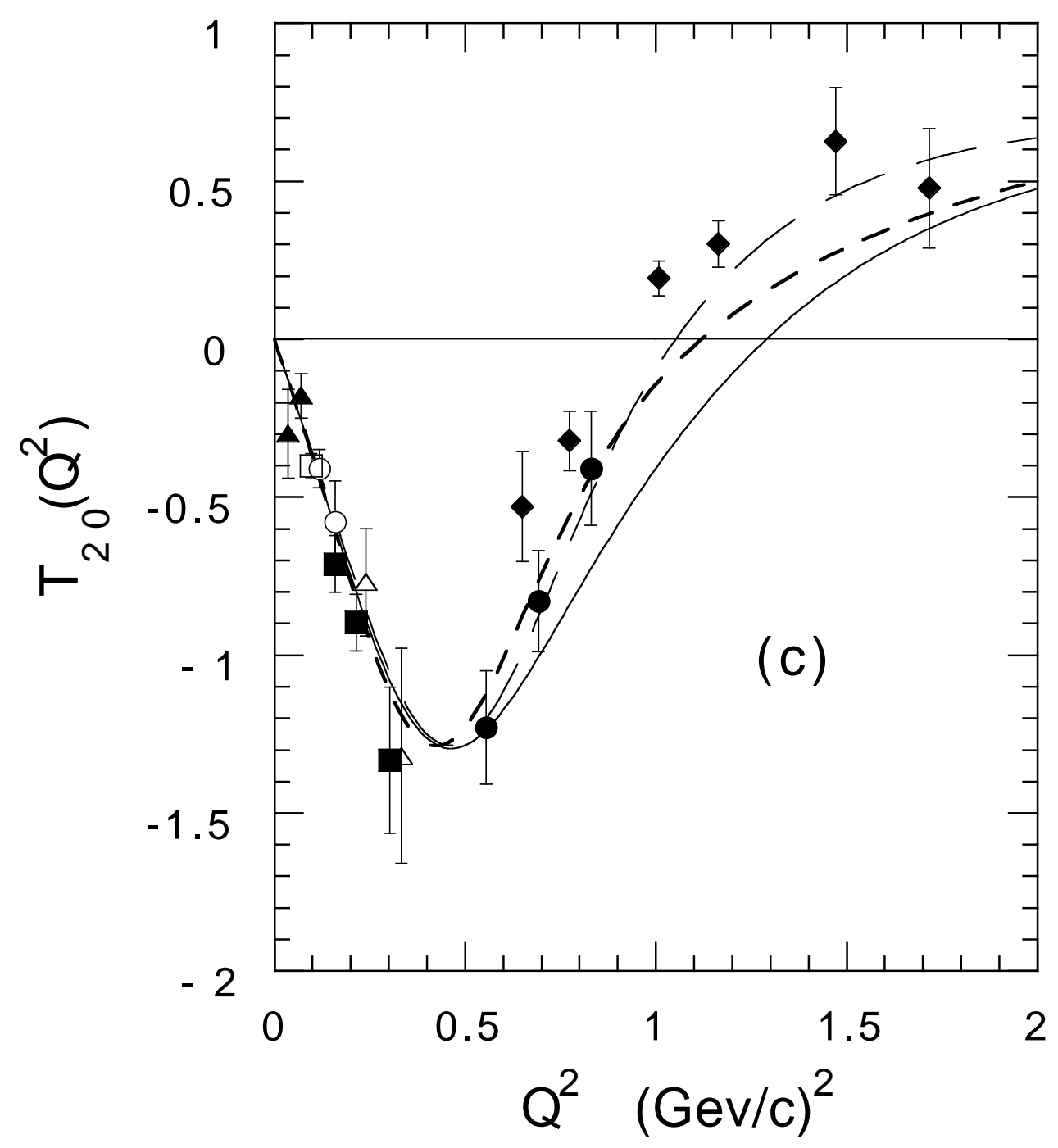

Fig. 1c F.M. LEV, E. PACE, G. SALMÈ 


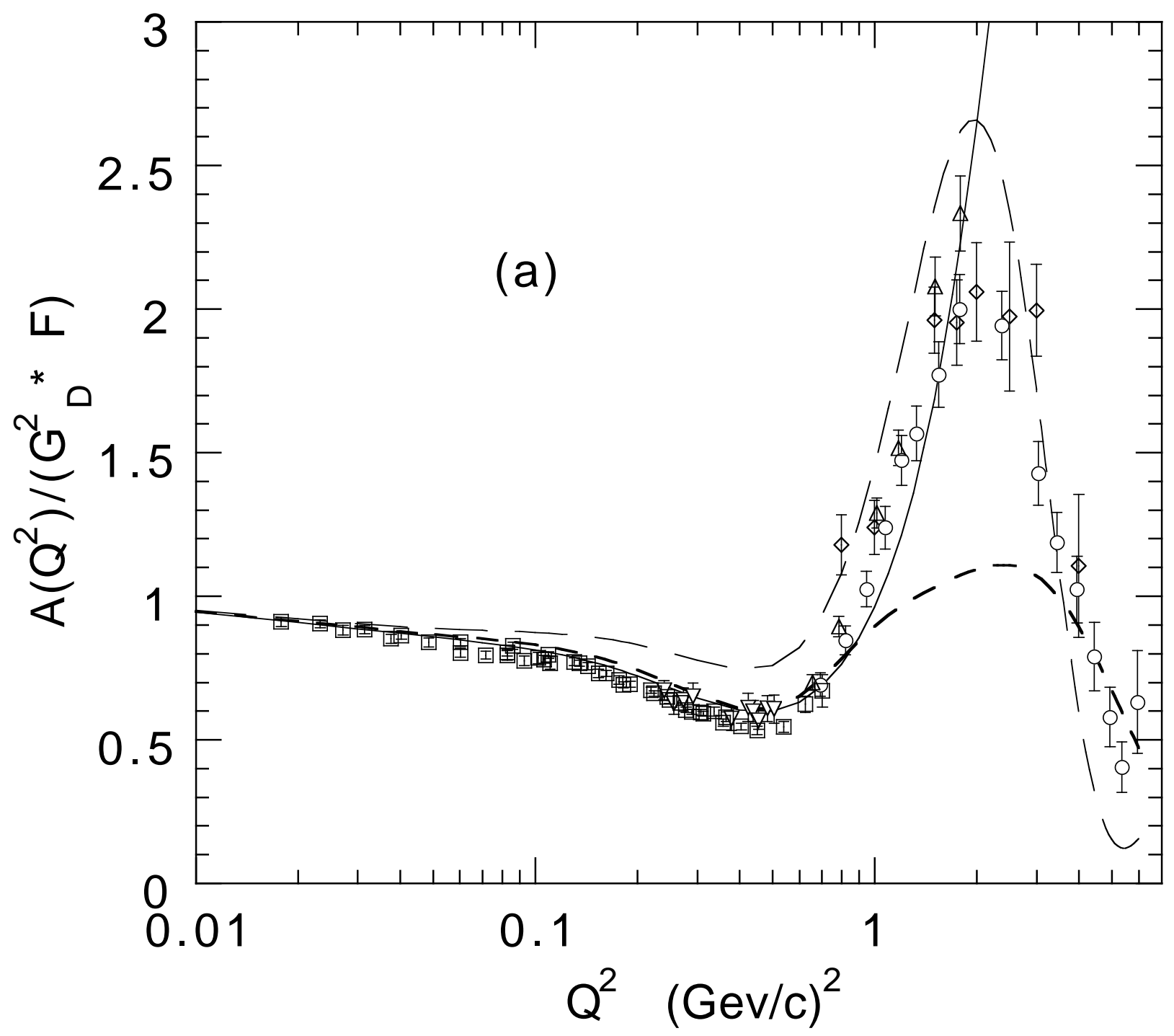

Fig. 2a F.M. LEV, E. PACE, G. SALMÈ 


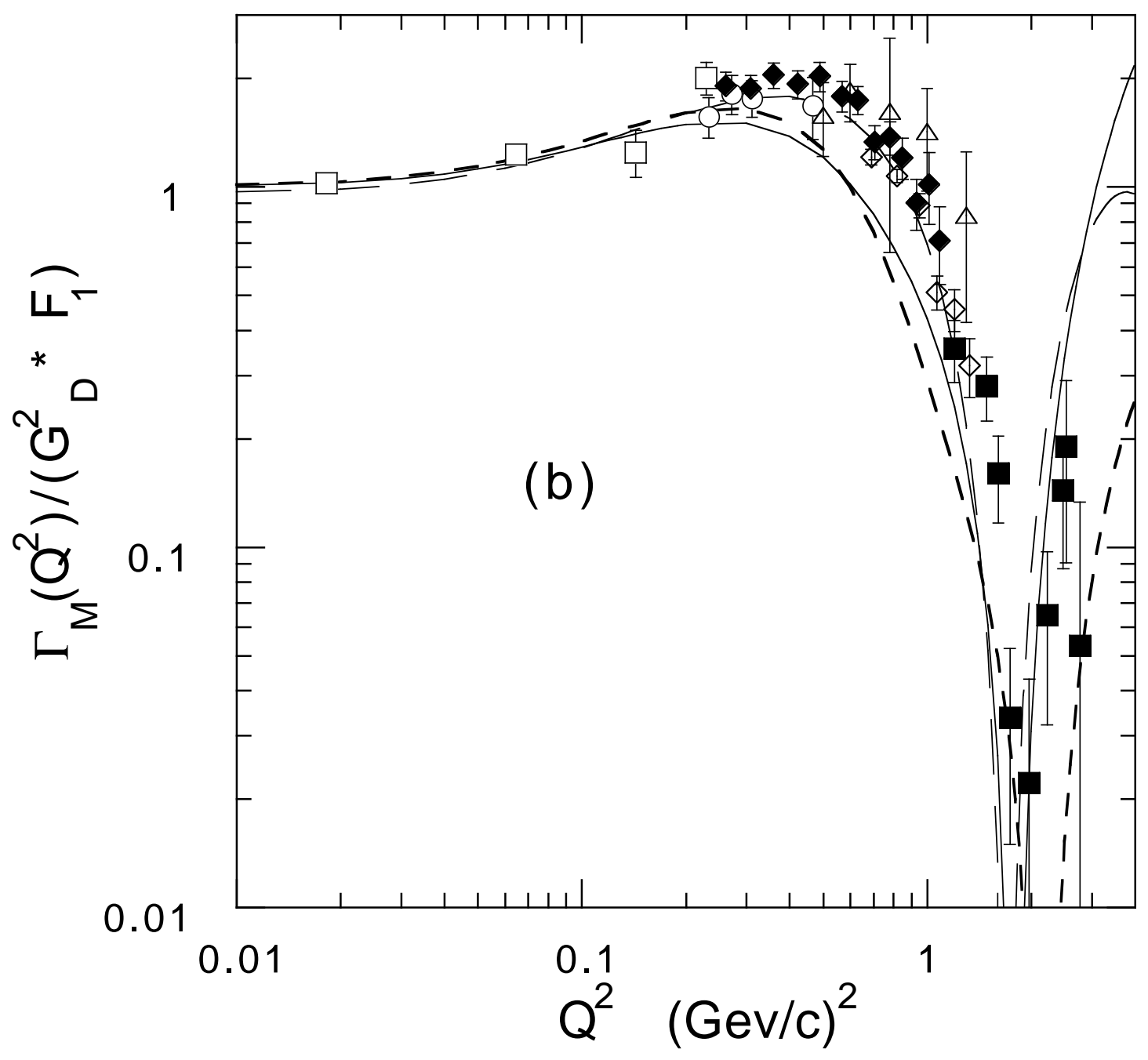

Fig. 2b F.M. LEV, E. PACE, G. SALMÈ 


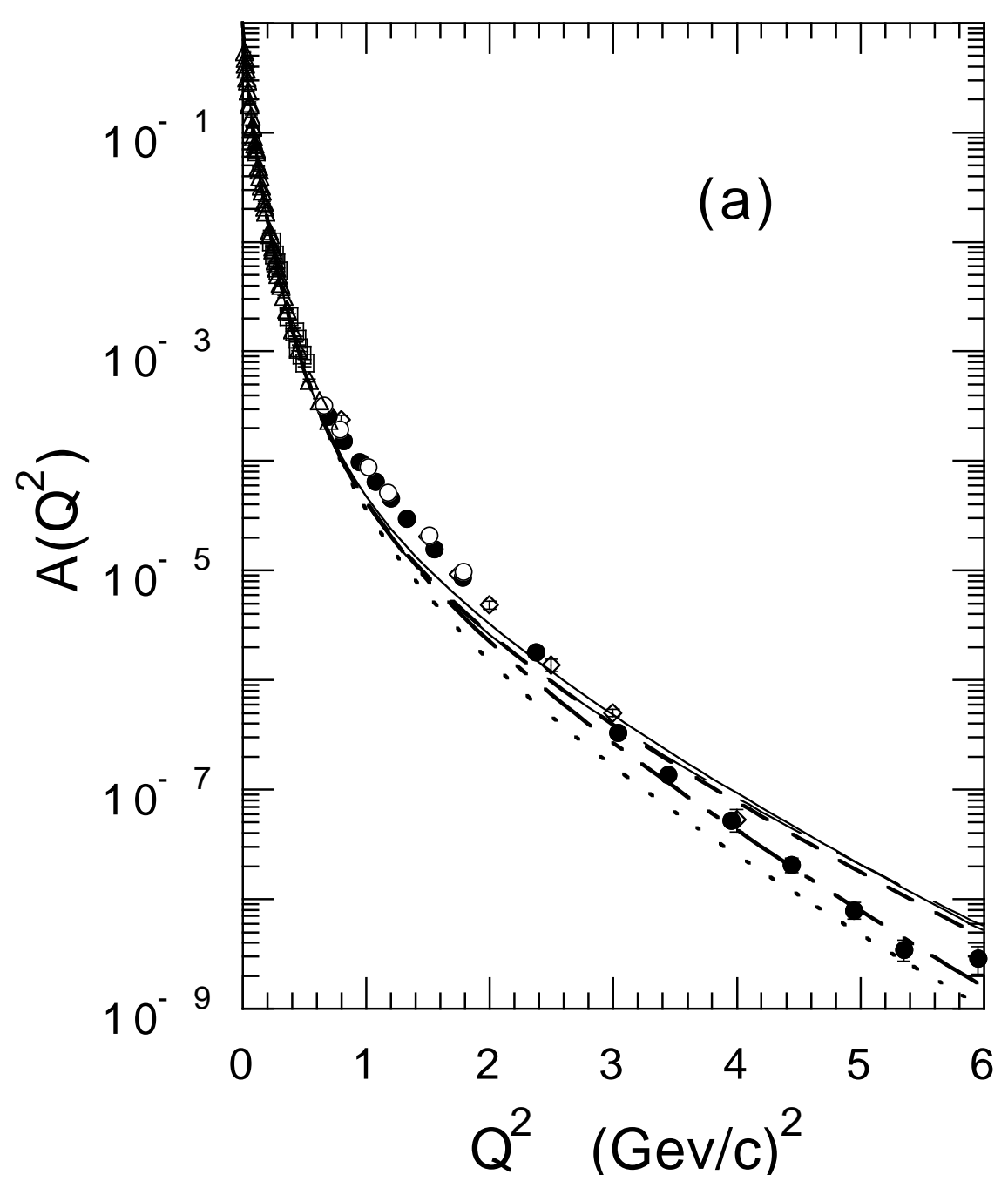

Fig. 3a F.M. LEV, E. PACE, G. SALMÈ 


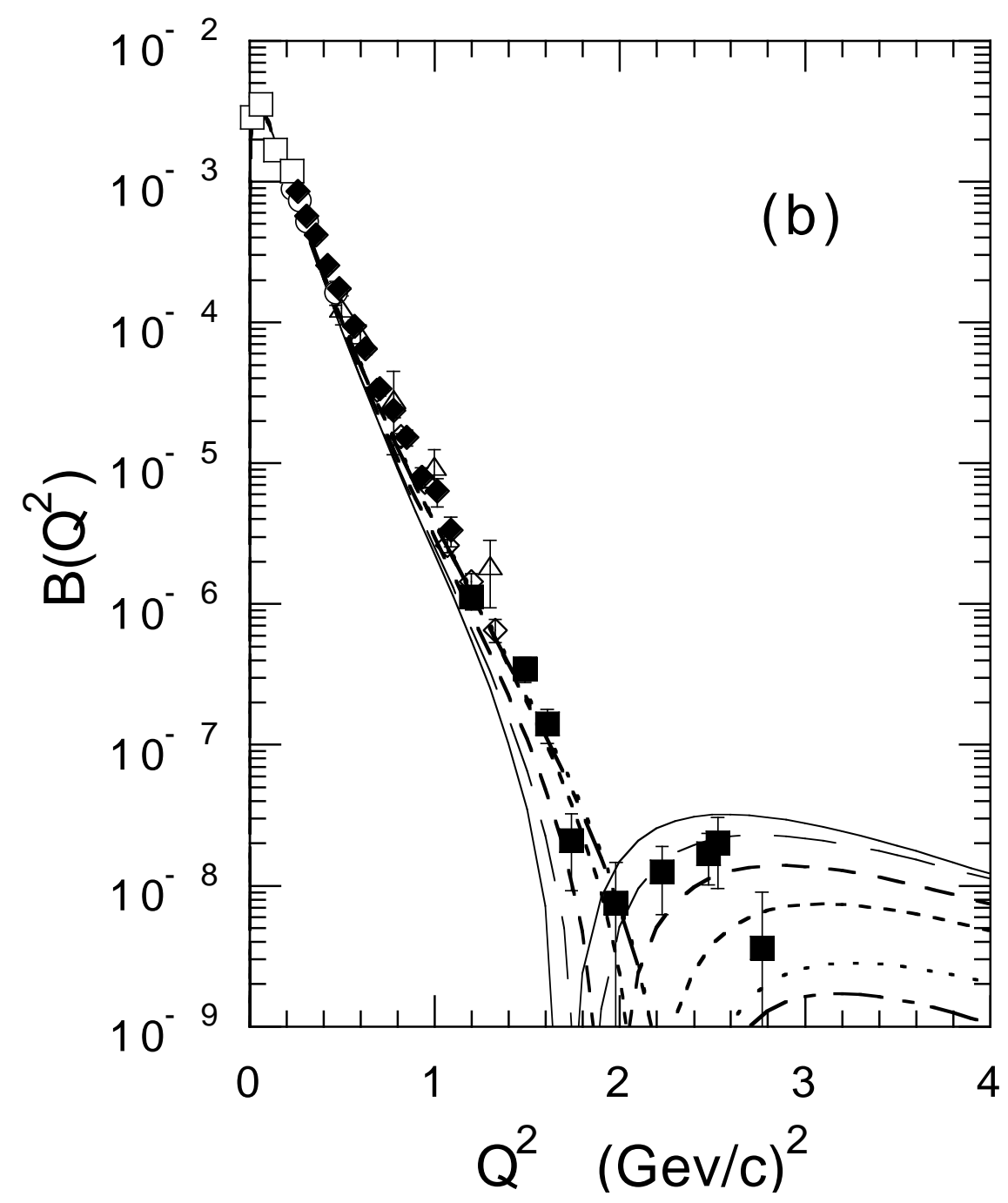

Fig. 3b F.M. LEV, E. PACE, G. SALMÈ 


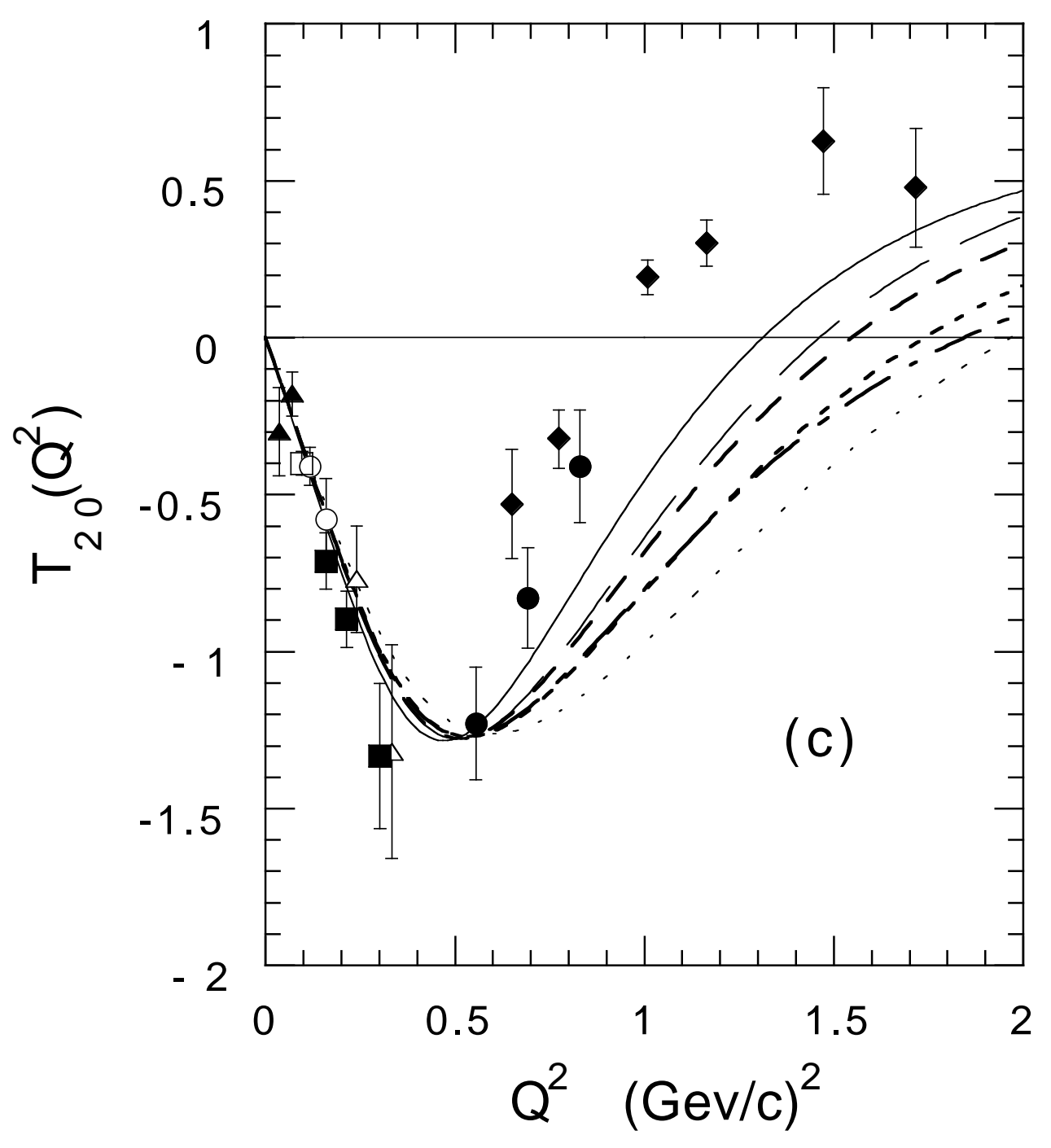

Fig. 3c F.M. LEV, E. PACE, G. SALMÈ 


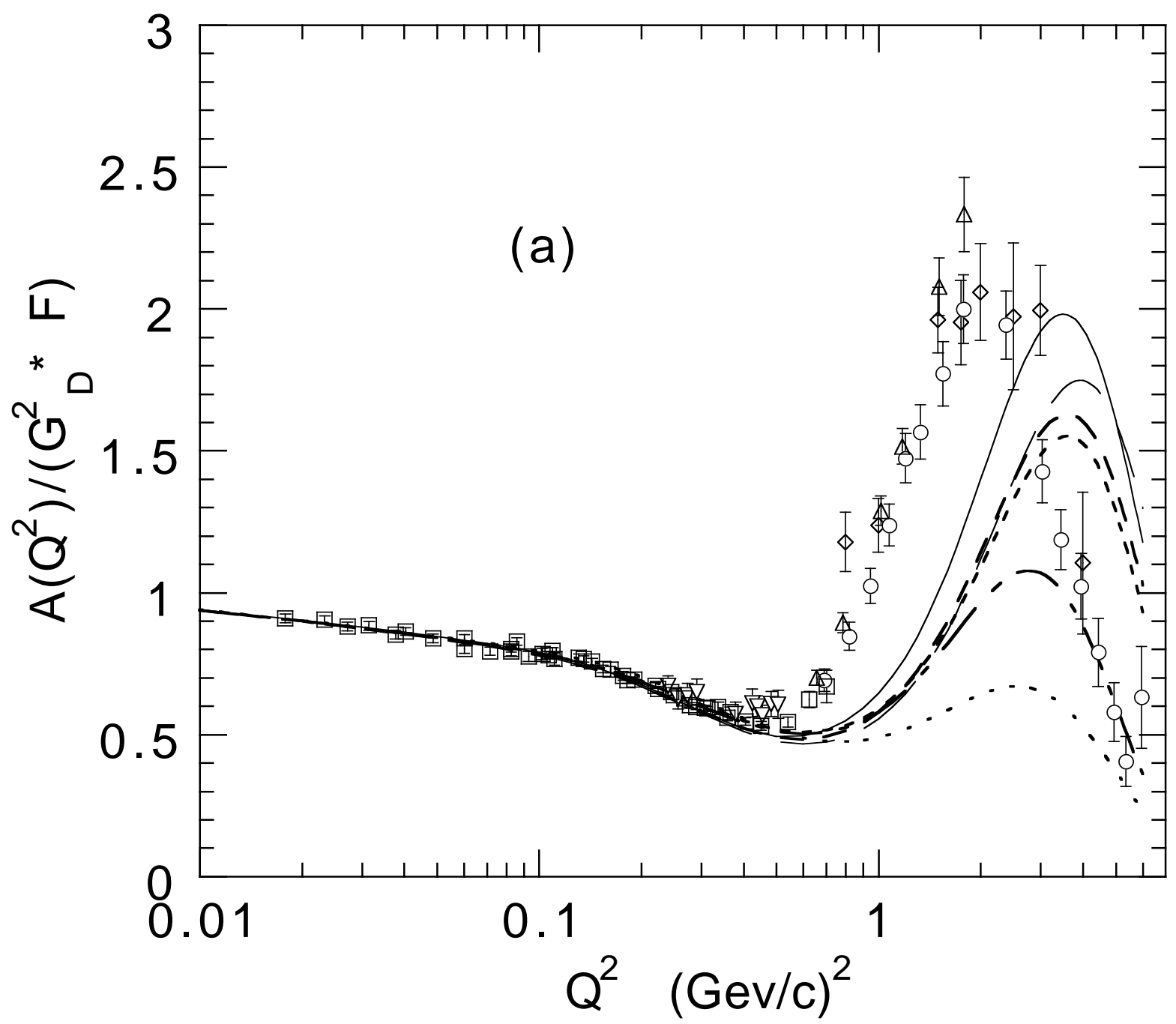

Fig. 4a F.M. LEV, E. PACE, G. SALMÈ 


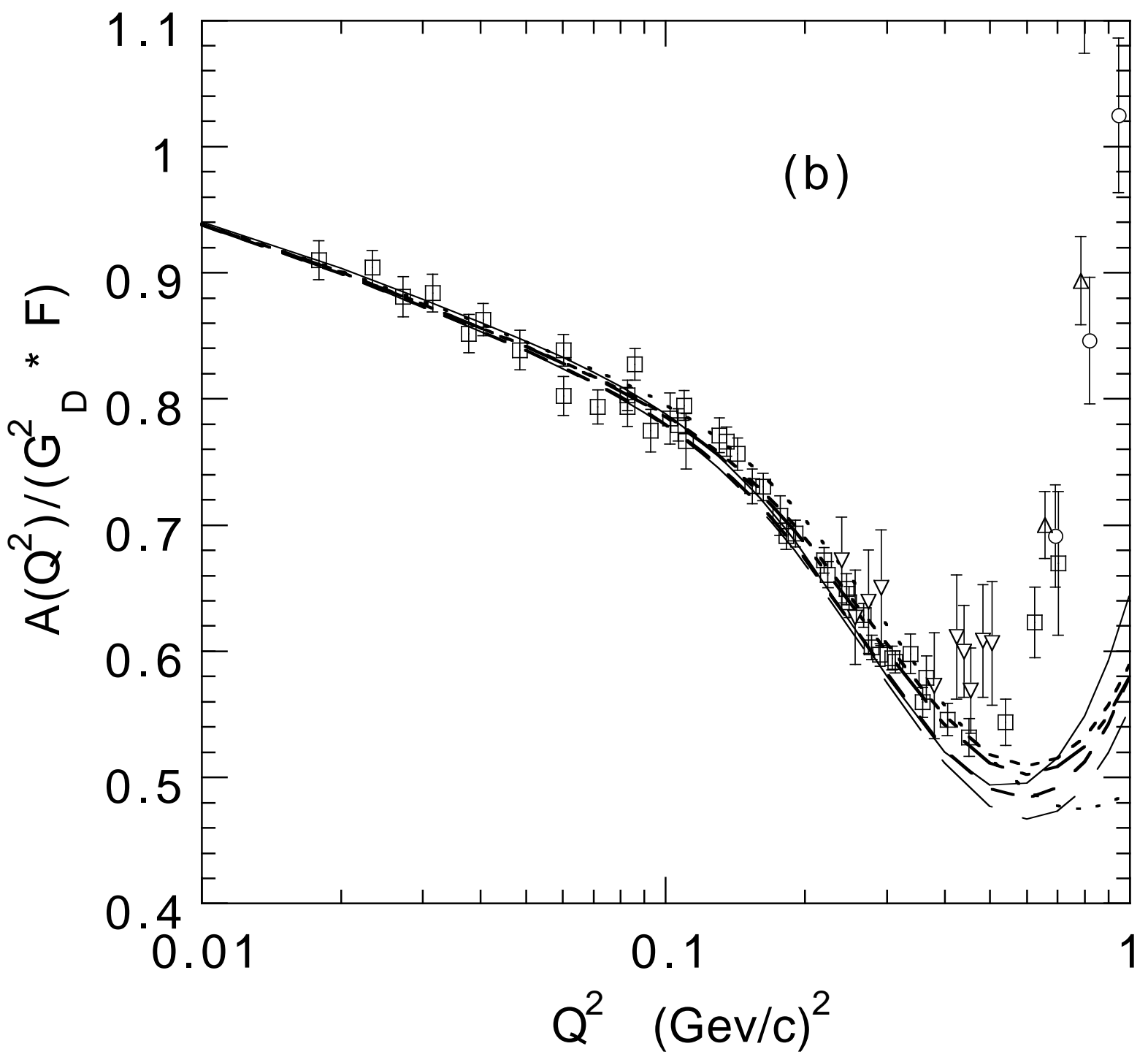

Fig. 4b F.M. LEV, E. PACE, G. SALMÈ 


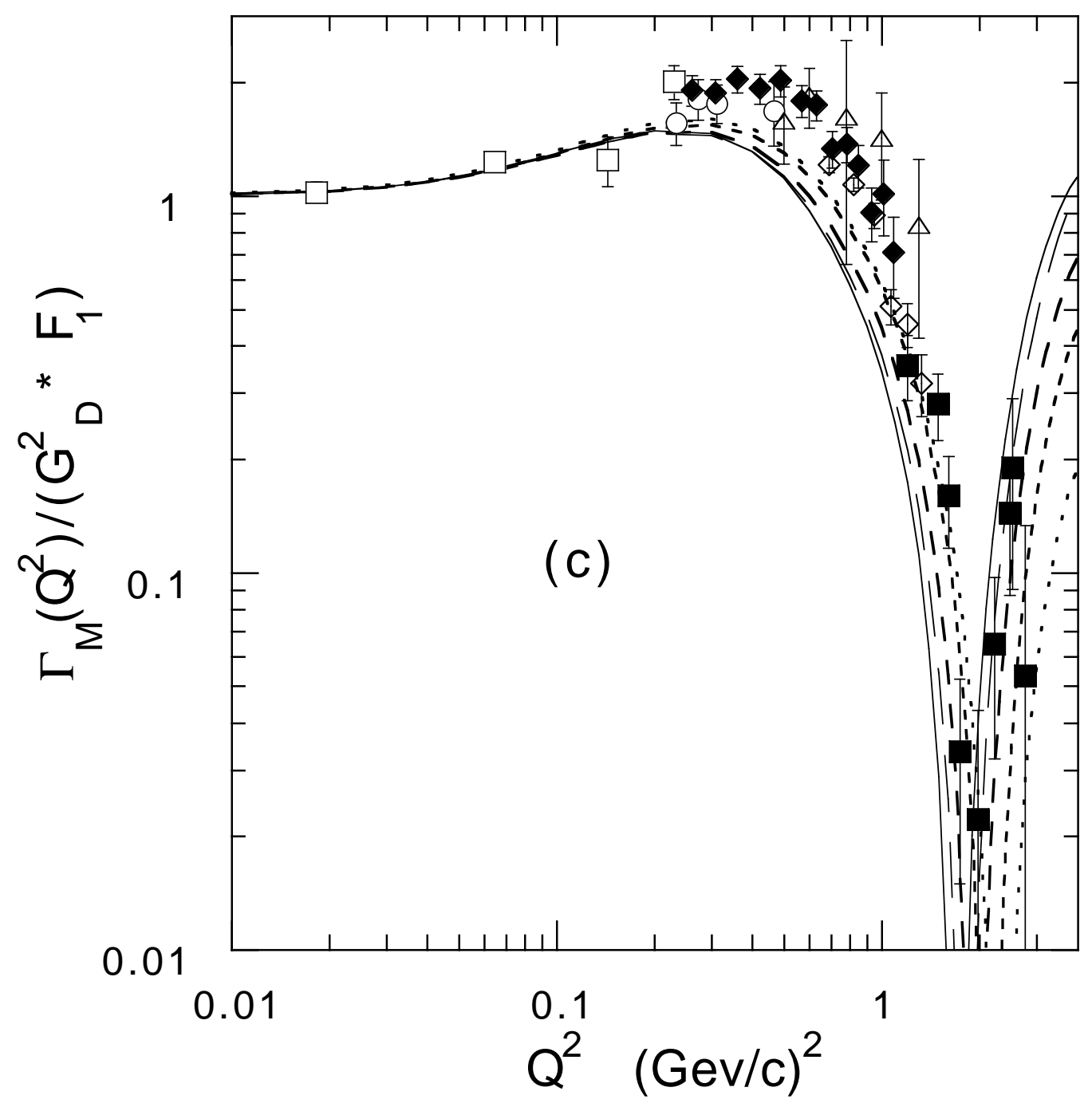

Fig. 4c F.M. LEV, E. PACE, G. SALMÈ 


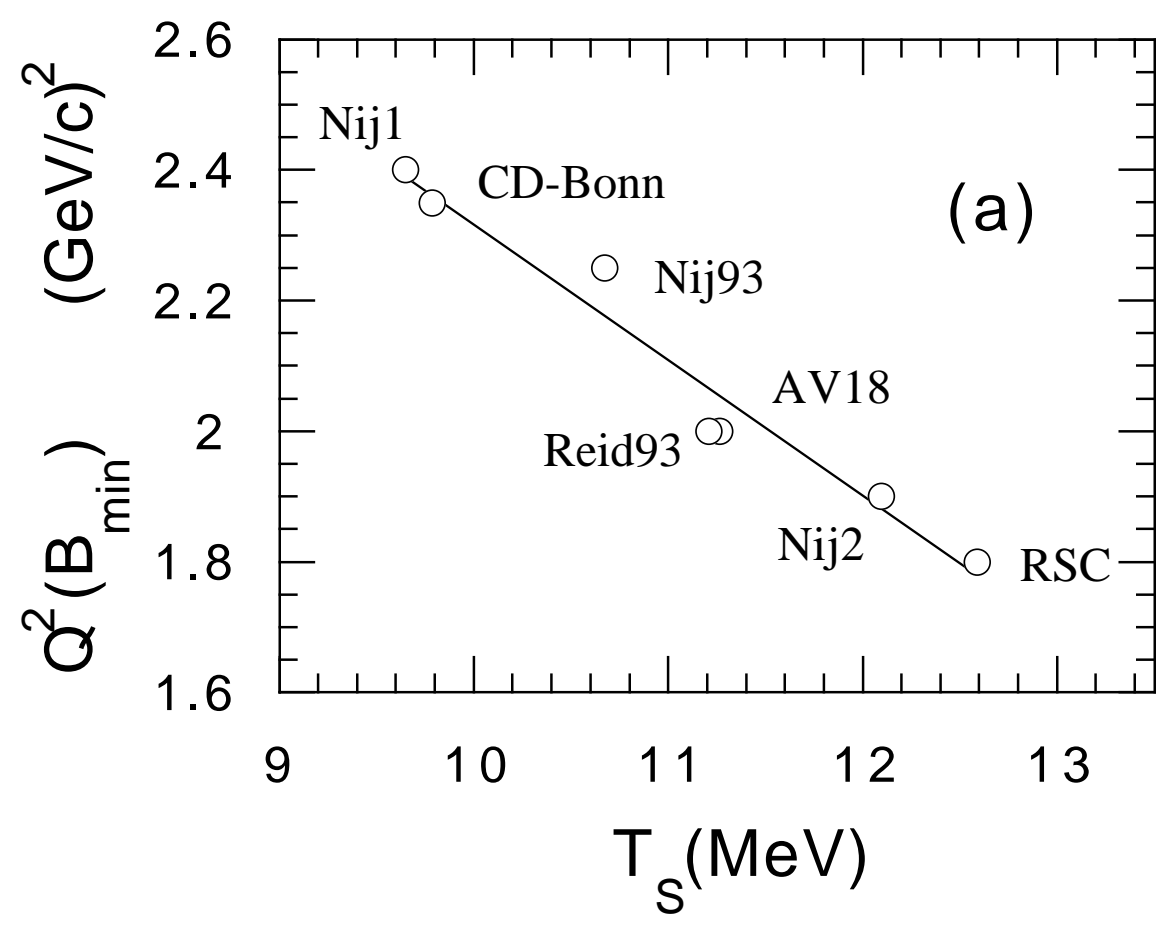

Fig. 5a F.M. LEV, E. PACE, G. SALMÈ 


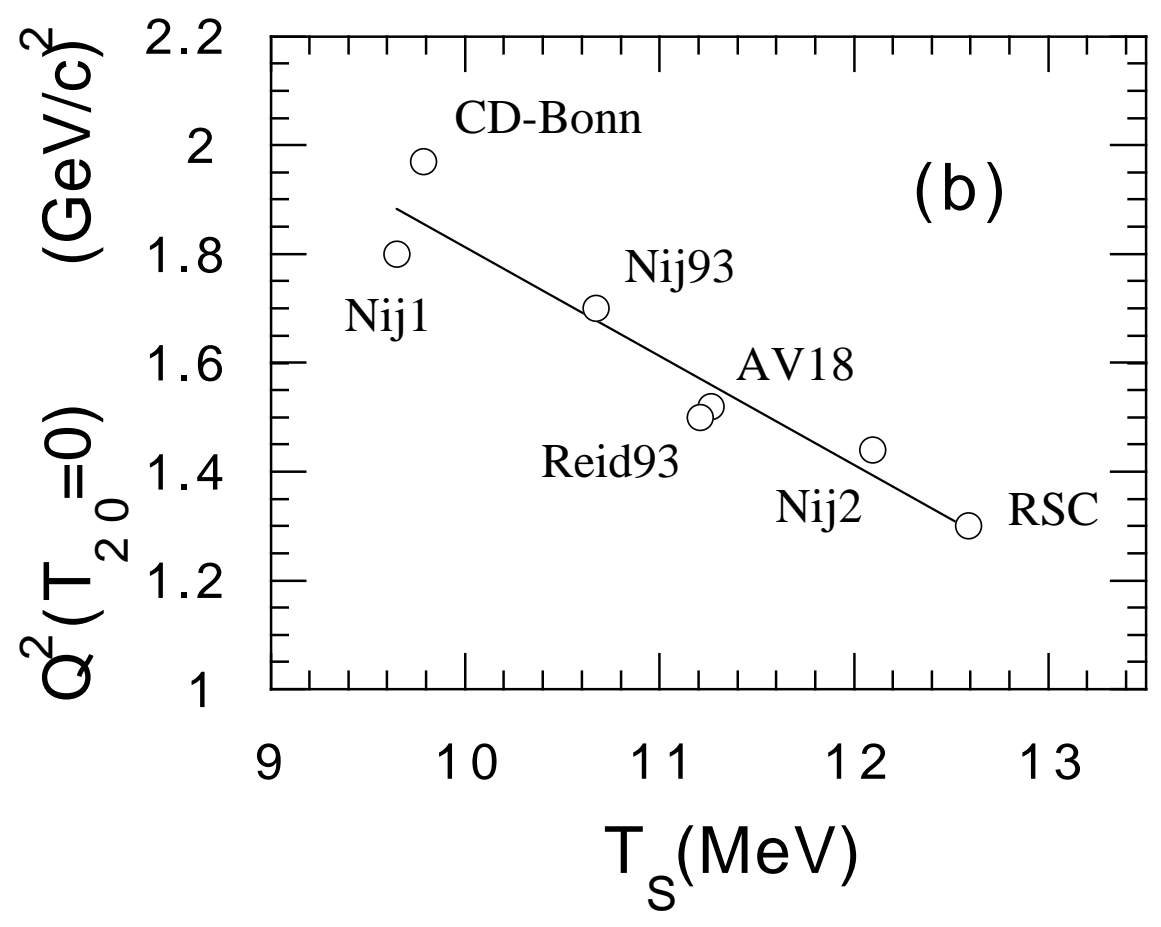

Fig. 5b F.M. LEV, E. PACE, G. SALMÈ 


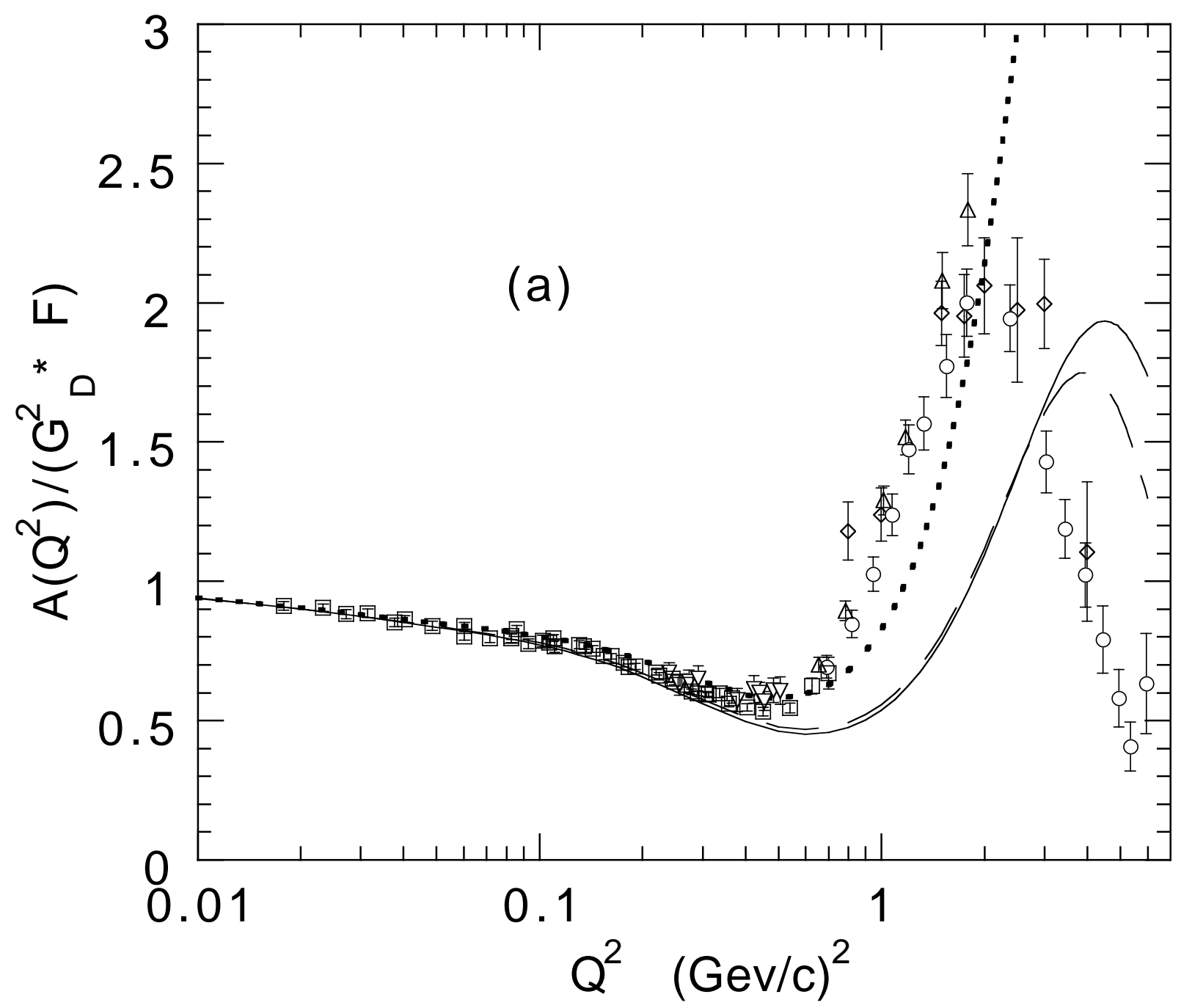

Fig. 6a F.M. LEV, E. PACE, G. SALMÈ 


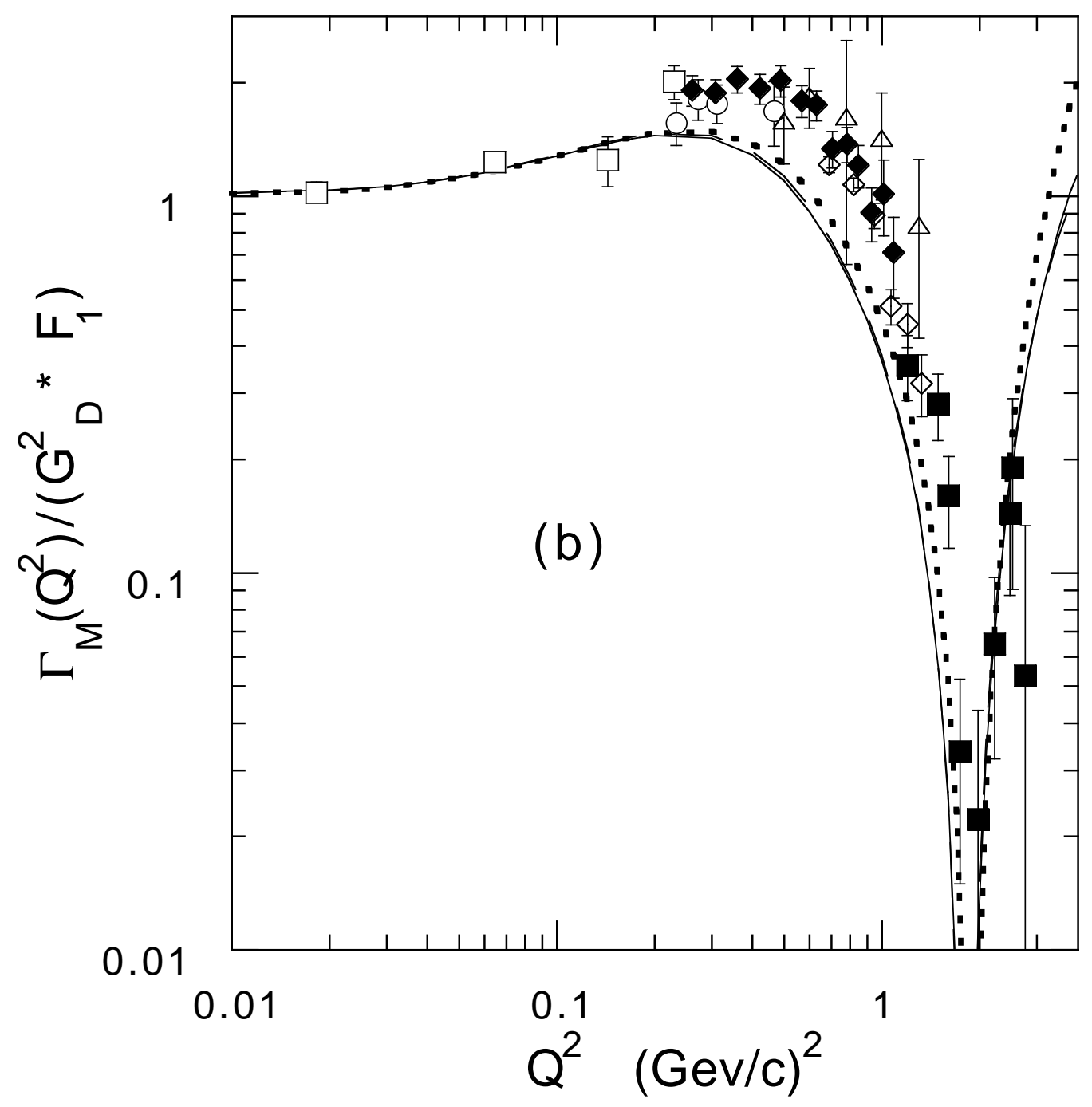

Fig. 6b F.M. LEV, E. PACE, G. SALMÈ 


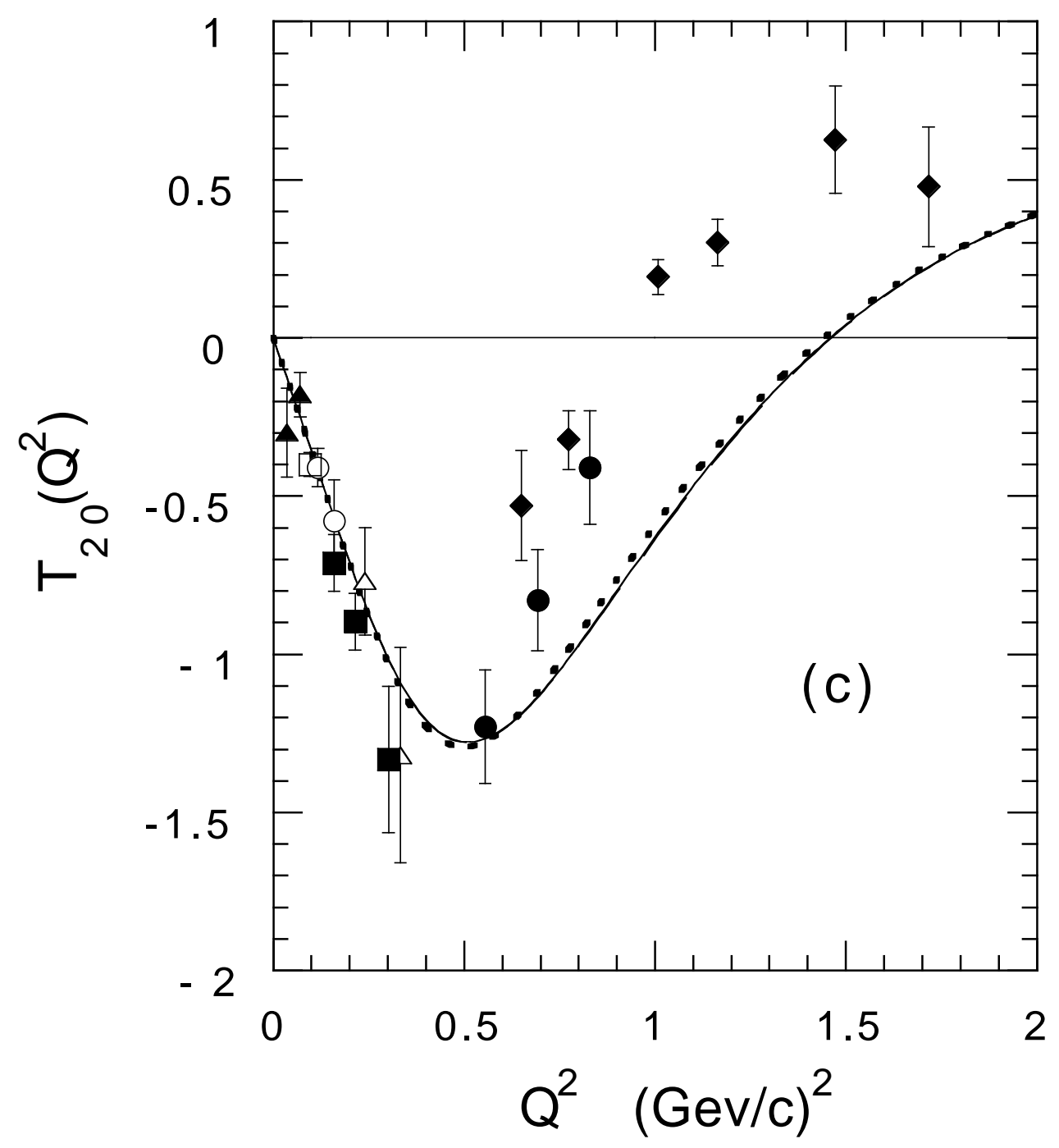

Fig. 6c F.M. LEV, E. PACE, G. SALMÈ 